\title{
Bergman-harmonic maps of balls
}

\author{
Elis abetTA BARletTA AND SORIN DRAGOMIR
}

\begin{abstract}
We study Bergman-harmonic maps between balls $\Phi: \mathbb{B}_{n} \rightarrow \mathbb{B}_{N}$ extending of class either $C^{2}$ or $\mathfrak{M}^{1}$ to the boundary of $\mathbb{B}_{n}$. For every holomorphic (anti-holomorphic) map $\Phi: \mathbb{B}_{n} \rightarrow \mathbb{B}_{N}$ extending smoothly to the boundary and every smooth homotopy $H: \Phi \simeq \Psi$ we prove a Lichnerowicz-type (cf. [28]) result, i.e., we show that $E_{\Omega_{\epsilon}}(\Psi) \geq E_{\Omega_{\epsilon}}(\Phi)+O\left(\epsilon^{-n+1}\right)$. When $\Phi$ is proper, Bergman-harmonic, and $C^{2}$ up to the boundary, the boundary values map $\phi: S^{2 n-1} \rightarrow S^{2 N-1}$ is shown to satisfy a compatibility system similar to the tangential Cauchy-Riemann equations on $S^{2 n-1}$ (and satisfied by the boundary values of any proper holomorphic map). For every weakly Bergman-harmonic map $\Phi \in W^{1}\left(\mathbb{B}_{n}, \mathbb{B}_{N}\right)$ admitting Sobolev boundary values $\phi \in \mathfrak{M}^{1}\left(S^{2 n-1}, \mathbb{B}_{N}\right)$ in the sense of [6], the boundary values $\phi$ are shown to be a weakly subelliptic harmonic map of $\left(S^{2 n-1}, \eta\right)$ into $\left(\mathbb{B}_{N}, h\right)$, provided that $\Phi^{-1} \nabla^{h}$ stays bounded at the boundary of $\mathbb{B}_{n}$ and $\phi$ has vanishing weak normal derivatives.
\end{abstract}

Mathematics Subject Classification (2010): 32H40 (primary); 32V20, 35H20, 35J20, 53C43, 58E20 (secondary).

\section{Statement of the main results}

We study maps $\Phi: \Omega \rightarrow D$ for smoothly bounded strictly pseudoconvex domains $\Omega \subset \mathbb{C}^{n}$ and $D \subset \mathbb{C}^{N}$, with an emphasis on the case $\Omega=\mathbb{B}_{n}$ and $D=\mathbb{B}_{N}$ where $\mathbb{B}_{n}=\left\{z \in \mathbb{C}^{n}:|z|<1\right\}$ is the unit ball, which are either strong or weak solutions to the Bergman-harmonic map system

$$
\tau_{g_{\Omega}}(\Phi)^{P} \equiv-\Delta_{g_{\Omega}} \Phi^{P}+\left(\Gamma_{Q R}^{P} \circ \Phi\right) \frac{\partial \Phi^{Q}}{\partial x^{a}} \frac{\partial \Phi^{R}}{\partial x^{b}} G^{a b}=0, \quad 1 \leq P \leq 2 N
$$

Here $\Omega$ and $D$ are thought of as endowed with the Bergman metric $g_{\Omega}$ and an $a$ priori arbitrary Riemannian metric $h$, respectively. There is an ample mathematical literature devoted to harmonic maps from the point of view of PDE theory $(c f ., e . g$., J. Jost [22] and R. Moser [29]) where the principal part in equations (1.1) is the

Received November 28, 2013; accepted in revised form April 17, 2014.

Published online February 2016. 
ordinary Laplacian (rather than the Bergman Laplacian $\Delta_{g_{\Omega}}$ ). We are interested in the regularity up to the boundary of the solution $\Phi$ to the Dirichlet problem $\tau_{g_{\Omega}}(\Phi)=0$ in $\Omega$ and $\Phi=\phi$ on $\partial \Omega$, supposing that $\Phi$ admits a trace on $\partial \Omega$ in a suitable sense. As far as $C^{\infty}$ regularity up to $\partial \Omega$ is concerned, the problem has been taken up by C. R. Graham [20,21], and by C. R. Graham and J. M. Lee [19], for scalar-valued maps $\Phi: \Omega \rightarrow \mathbb{R}$. As ellipticity of $\Delta_{g_{\Omega}}$ degenerates at the boundary, new phenomena were shown to arise (cf. op. cit.) i.e. the existence of a solution $\Phi \in C^{\infty}(\bar{\Omega}, \mathbb{R})$ to the Dirichlet problem $\Delta_{g_{\Omega}} \Phi=0$ in $\Omega$ and $\Phi=\phi$ on $\partial \Omega$ requires the boundary datum $\phi \in C^{\infty}(\partial \Omega, \mathbb{R})$ to satisfy certain compatibility equations $\mathcal{C}(\phi)=0$ along $\partial \Omega$. By an elementary result in differential geometry, any holomorphic map between Kählerian manifolds is harmonic. In particular any holomorphic map $\Phi: \Omega \rightarrow D$ is harmonic as a map of $\left(\Omega, g_{\Omega}\right)$ into $(D, h)$, provided that $h$ is a Kählerian metric. By a result of A. Lichnerowicz, [28], if the source and target Kählerian manifolds are compact then any holomorphic map is actually a stable harmonic map and an absolute minimum in its homotopy class. A. Lichnerowicz's result fails for holomorphic maps of balls $\Phi: \mathbb{B}_{n} \rightarrow \mathbb{B}_{N}$, due to the lack of compactness. How much of A. Lichnerowicz's approach does survive for maps between balls? As an attempt to answer the previous question we obtain the following result (deferring definitions to the next sections)

Theorem 1.1. Let $\Phi: \mathbb{B}_{n} \rightarrow \mathbb{B}_{N}$ be a $C^{2}$ map. For every domain $\Omega \Subset \mathbb{B}_{n}$ and every smooth homotopy $H: \mathbb{B}_{n} \times[0,1] \rightarrow \mathbb{B}_{N}$ such that $H_{0}=\Phi$ we set

$$
a_{M}(H)=\int_{M} i_{M}^{*}\left\{\alpha(H) \wedge * \mathcal{W}^{\mathbb{B}_{n}}\right\}
$$

where $M=\partial \Omega$ and $i_{M}: M \rightarrow \mathbb{B}_{n}$ is the inclusion.

i) If $\Phi \in \operatorname{Hol}_{ \pm}(n, N)$ then

$$
E_{\Omega}(\Psi) \geq\left|E_{\Omega}(\Phi) \pm a_{M}(H)\right|
$$

where $\Psi=H_{1}: \mathbb{B}_{n} \rightarrow \mathbb{B}_{N}$.

ii) If $\Psi \in \operatorname{Hol}_{ \pm}(n, N)$ too, then $E_{\Omega}(\Phi) \geq\left|E_{\Omega}(\Psi) \mp a_{M}(H)\right|$. Consequently if $a_{M}(H)=0$ for some $H: \Phi \simeq \Psi$ then $a_{M}(H)=0$ for all.

iii) Let $H$ be a smooth homotopy such that

$$
\left(\left(g_{\mathbb{B}_{N}}\right)_{A \bar{B}} \circ H\right) \frac{\partial H^{A}}{\partial t} \frac{\partial H_{\bar{B}}}{\partial z^{j}} z^{j}, \quad\left(\left(g_{\mathbb{B}_{N}}\right)_{A \bar{B}} \circ H\right) \frac{\partial H^{A}}{\partial t} \frac{\partial H_{\bar{B}}}{\partial \bar{z}_{j}} \bar{z}_{j},
$$

stay bounded as $|z| \rightarrow 1$. Then

$$
\left|a_{M_{\epsilon}}(H)\right| \leq \epsilon^{-(n-1)} C_{n}\|u\|_{L^{\infty}} \operatorname{Vol}\left(M_{\epsilon}, i_{\epsilon}^{*} \theta\right)
$$

where $i_{\epsilon}: M_{\epsilon}=S^{2 n-1}\left(\sqrt{1-\epsilon / a_{n}}\right) \hookrightarrow \mathbb{B}_{n}$ with $a_{n}=\left(\pi^{n} / n !\right)^{1 /(n+1)}$ and $u=\alpha(H) T$ and $C_{n}=2^{n+1}(n+1)^{n+1} /(n-1)$ ! In particular $a_{M_{\epsilon}}(H)=$ $O\left(\epsilon^{-n+1}\right)$ as $\epsilon \rightarrow 0$. 
So unlike the case in [28] (where $\Omega$ is the whole source manifold and the above boundary integral vanishes) one only has asymptotic information on $\int_{M_{\epsilon}} u \Psi_{\theta}$ as $\epsilon \rightarrow 0$, prompting the study of Bergman-harmonic maps $\Phi: \Omega \rightarrow D$, extending to the boundary, through the mathematical analysis of their boundary values. Our findings in the smooth regularity up to the boundary problem are contained in the following ( $c f$. Section 6)

Theorem 1.2. Let $\Phi: \Omega \rightarrow D$ be a Bergman-harmonic map of a strictly pseudconvex domain $\Omega \subset \mathbb{C}^{n}$ endowed with the Bergman metric $g_{\Omega}$, into a domain $D \subset \mathbb{C}^{N}$ endowed with a Riemannian metric $h$.

1) Assume that:

i) $\lim _{\Omega \ni z \rightarrow z_{0}} \Phi(z) \in D$ i.e. the limit exists and belongs to $D$;

ii) $\lim _{\Omega \ni z \rightarrow z_{0}} \Gamma_{Q R}^{P}(\Phi(z))=\Gamma_{Q R}^{P}\left(\Phi\left(z_{0}\right)\right)$ for every boundary point $z_{0} \in \partial \Omega$.

Let $\phi: \partial \Omega \rightarrow D$ be the boundary values of $\Phi$. Then

$$
N\left(\phi^{P}\right)=-\frac{1}{2(n-1)} \tau_{b}(\phi)^{P}, \quad 1 \leq P \leq 2 N .
$$

In particular if $\phi$ has vanishing normal derivatives then $\phi$ is subelliptic harmonic as a map from the pseudohermitian manifold $(\partial \Omega, \theta)$ into the Riemannian manifold $(D, h)$.

2) Let $D=\mathbb{B}_{N}$ and let $h=g_{D}$ be its Bergman metric. Assume that $\Phi$ is proper and extends smoothly up to the boundary of $\Omega$. Then the boundary values map $\phi=\left(\phi^{1}, \ldots, \phi^{N}\right): \partial \Omega \rightarrow S^{2 N-1}$ satisfies

$$
\sum_{A=1}^{N} \phi_{\bar{A}} \bar{\partial}_{b} \phi^{A}=0
$$

where $\bar{\partial}_{b}$ is the tangential Cauchy-Riemann operator on $\partial \Omega$.

Aside from the technical difficulties arising from allowing more general values in the Riemannian manifold $(D, h)$, and from dealing with the nonlinear system of PDEs (1.1) rather than the single linear PDE $\Delta_{g_{\Omega}} \Phi=0$, Theorem 1.2 is in spirit rather similar to the result in [19] (and to prove it we make use of the same geometric approach employed there, relying on the Graham-Lee connection, followed by an elementary asymptotic analysis towards $\partial \Omega$ ). A methodologically new result is obtained in the weakly differentiable case, where we only assume that $\Phi: \mathbb{B}_{n} \rightarrow$ $\mathbb{B}_{N}$ is a Sobolev map, admitting boundary values in the sense of G. Cimmino [9]. To make sense of $L^{p}$-boundary values for a function $u: \Omega \rightarrow \mathbb{R}$ one sets $u_{\lambda}: \partial \Omega \rightarrow$ $\mathbb{R}, u_{\lambda}=u \circ \delta_{\lambda}$, which requires the domain $\Omega$ to be $\delta_{\lambda}$-contractible i.e. $\delta_{\lambda}(\partial \Omega) \subset \Omega$ for every $0<\lambda<1$, where $\delta_{\lambda}(z)=\lambda z$ for any $z \in \partial \Omega$. Successively, given $\varphi \in L^{p}(\partial \Omega)$, one requires that $u_{\lambda} \rightarrow \varphi$ in $L^{p}(\partial \Omega)$ as $\lambda \rightarrow 1^{-} . C f .[6,26]$, where this approach is taken in order to formulate and solve the Dirichlet problem with $L^{p}$-boundary data for a class of sublaplacians. It is interesting to note that none of 
G. Cimmino's works ( $c f . e . g . ~[10])$ is reported on in the classical monograph [18], and the approach to the generalized solution $u \in W^{1}(\Omega)$ to the Dirichlet problem for a second order elliptic operator is to assume that the boundary datum $\varphi$ admits one $L^{2}$ weak derivative, i.e. $\varphi \in W^{1}(\Omega)$, a case in which the boundary condition $u=\varphi$ may be written merely as $u-\varphi \in W_{0}^{1}(\Omega)(c f$. [18, Theorem 8.3, page 181]. Our result is ( $c f$. Section 7):

Theorem 1.3. Let $\Phi \in W^{1}\left(\mathbb{B}_{n}, \mathbb{B}_{N}\right)$, with $n \geq 3$, be a weakly Bergman-harmonic map admitting $L^{2}$ boundary values $\phi \in L^{2}\left(S^{2 n-1}, \mathbb{R}^{2 N}\right)$. Assume further that $\phi \in \mathfrak{M}^{1}\left(S^{2 n-1}, \mathbb{R}^{2 N}\right)$ and $\Phi_{\lambda} \rightarrow \phi$ in $\mathfrak{M}^{1}\left(S^{2 n-1}, \mathbb{R}^{2 N}\right)$ as $\lambda \rightarrow 1^{-}$.

a) Suppose that:

i) $\phi \in \mathfrak{M}^{1}\left(S^{2 n-1}, \mathbb{B}_{N}\right)$ i.e. $\phi(z) \in \mathbb{B}_{N}$ for a.e. $z \in S^{2 n-1}$;

ii) the Kählerian metric $h$ on $\mathbb{B}_{N}$ satisfies $\Gamma_{Q R}^{P} \circ \Phi_{\lambda} \rightarrow \Gamma_{Q R}^{P} \circ \phi$ in $L^{2}\left(S^{2 n-1}\right)$ as $\lambda \rightarrow 1^{-}$for any $1 \leq P, Q, R \leq 2 N$.

Then $\phi$ is a weakly subelliptic harmonic map of the pseudohermitian manifold $\left(S^{2 n-1}, \eta\right)$ into the Riemannian manifold $\left(\mathbb{B}_{N}, h\right)$.

b) Suppose that:

iii) $h=g_{\mathbb{B}_{N}}$;

iv) $\phi \in \mathfrak{M}^{1}\left(S^{2 n-1}, S^{2 N-1}\right)$ i.e. $\phi(z) \in S^{2 N-1}$ for almost every $z \in S^{2 n-1}$;

v) there is $Q_{\phi} \in L^{2}\left(S^{2 n-1}\right)$ such that

$$
\lim _{\lambda \rightarrow 1^{-}} \frac{1-\left|\Phi_{\lambda}\right|^{2}}{1-\lambda^{2}}=Q_{\phi}
$$

in $L^{2}\left(S^{2 n-1}\right)$ and $Q_{\phi}(\zeta) \neq 0$ for a.e. $\zeta \in S^{2 n-1}$.

Then

$$
\phi_{\bar{A}} \bar{\partial}_{b} \phi^{A}=0, \quad 1 \leq A \leq N
$$

a.e. in $S^{2 n-1}$.

c) Any weakly $C R$ map $\phi: S^{2 n-1} \rightarrow S^{2 N-1}$ is a solution to (1.7).

To make sense of the compatibility equations, the boundary datum is required to possess one weak derivative along $H\left(S^{2 n-1}\right)$ in $L^{2}\left(S^{2 n-1}, \mathbb{R}^{2 N}\right.$ ) (and use is made of the Sobolev type spaces $\mathfrak{M}^{1}\left(S^{2 n-1}, \mathbb{R}^{2 N}\right)$ whose local counterparts, for an open subset $U \subset S^{2 n-1}$ carrying a local frame of $H\left(S^{2 n-1}\right)$, are familiar from the theory of Hörmander systems of vector fields, $c f$. e.g. [34]). When $h=g_{\mathbb{B}_{N}}$ we need the additional assumption (1.6) that the quotient $\left(Q_{\Phi}\right)_{\lambda}$ (amply made use of in [32] and [11-13]) has a $L^{2}$ limit as $\lambda \rightarrow 1^{-}$. All proper holomorphic maps $\Phi: \mathbb{B}_{2} \rightarrow \mathbb{B}_{3}$ in J. J. Faran's list ( $c f$. [16]) may be seen to possess this property. The main novelty consists in the asymptotic analysis of the weakly Bergman-harmonic map system (7.1) towards the boundary, leading to the weakly subelliptic harmonic map system (2.2). $C f$. our Section 7 where we also explain the meaning of assumption (v) in Theorem 1.3. 
ACKNOWLEDGEMENTS. This paper was partially written while S. Dragomir visited at University of Illinois at Urbana-Champaign (April 4-30, 2013). He is grateful for invaluable discussions with John P. D'Angelo and for the hospitality and excellent working conditions at the Department of Mathematics of UIUC. E. Barletta and S. Dragomir acknowledge support from Italian government through P.R.I.N. 2012 Varietà Reali e Complesse: Geometria, Topologia e Analisi Armonica. The Authors are grateful to the Referee for his/her many corrections.

\section{Calculus on pseudohermitian manifolds and subelliptic harmonic maps}

Subelliptic harmonic maps were first studied by J. Jost and C-J. Xu [23] (to whom the notion is due) and Z-R. Zhou [36], within the theory of Hörmander systems of vector fields ( $c f$. e.g. A. Bonfiglioli et al. [7]) and were soon recognized ( $c f$. E. Barletta et al. [3]) to be local manifestations of pseudoharmonic maps from a pseudohermitian manifold into a Riemannian manifold ( $c f$. also [15, Chapter IV]). Precisely, let $\left(M, T_{1,0}(M)\right)$ be an oriented CR manifold, of CR dimension $n$, where $T_{1,0}(M) \subset T(M) \otimes \mathbb{C}$ denotes its CR structure. Let $H(M)=\operatorname{Re}\left\{T_{1,0}(M) \oplus T_{0,1}(M)\right\}$ be the Levi distribution, where $T_{0,1}(M)=\overline{T_{1,0}(M)}$ (here an overbar denotes complex conjugation). It carries the complex structure $J: H(M) \rightarrow H(M)$ given by $J(Z+\bar{Z})=i(Z-\bar{Z})$ for every $Z \in T_{1,0}(M)$. Let $\eta$ be a pseudohermitian structure on $M$ i.e. a globally defined nowhere vanishing $C^{\infty}$ section in the conormal bundle $H(M)^{\perp} \subset T^{*}(M)$, where $H(M)_{x}^{\perp}=\left\{\omega \in T_{x}^{*}(M): \operatorname{Ker}(\omega) \supset H(M)_{x}\right\}$ for every $x \in M$. The Levi form is $G_{\eta}(X, Y)=(d \eta)(X, J Y)$ for any $X, Y \in H(M)$. The CR manifold $M$, or its CR structure $T_{1,0}(M)$, is nondegenerate (strictly pseudoconvex) if $G_{\eta}$ is nondegenerate (positive definite) for some $\eta$. If $M$ is nondegenerate then every pseudohermitian structure $\eta$ is a contact form i.e. $\Psi_{\eta}=\eta \wedge(d \eta)^{n}$ is a volume form on $M$. The divergence operator $\operatorname{div} \equiv \operatorname{div}_{\eta}: \mathfrak{X}(M) \rightarrow C^{\infty}(M)$ is then given by

$$
\mathcal{L}_{X} \Psi_{\eta}=\operatorname{div}(X) \Psi_{\eta}, \quad X \in \mathfrak{X}(M) .
$$

Also there is a unique tangent vector field $T \in \mathfrak{X}(M)$ (the Reeb field of $(M, \eta)$ ) such that $\eta(T)=1$ and $(d \eta)(T, \cdot)=0$. As

$$
T(M)=H(M) \oplus \mathbb{R} T
$$

the Levi form $G_{\eta}$ extends to a semi-Riemannian metric $g_{\eta}$ (the Webster metric of $(M, \eta))$ determined by

$$
g_{\eta}(X, Y)=G_{\eta}(X, Y), \quad g_{\eta}(X, T)=0, \quad g_{\eta}(T, T)=1,
$$

for any $X, Y \in H(M)$. Once a contact form $\eta$ is fixed, there is a unique linear connection $\nabla$ on $M$ (the Tanaka-Webster connection of $(M, \eta)$ ) such that i) $H(M)$ is $\nabla$ parallel, $\nabla J=0$ and $\nabla g_{\eta}=0$, ii) $T_{\nabla}(Z, W)=0$ and $T_{\nabla}(Z, \bar{W})=2 i G_{\eta}(Z, \bar{W}) T$ for any $Z, W \in T_{1,0}(M)$, iii) $\tau \circ J+J \circ \tau=0$. Cf. e.g. [15], Chapter I. Here $T_{\nabla}$ is 
the torsion tensor field of $\nabla$ and $\tau(X)=T_{\nabla}(T, X)$ (the pseudohermitian torsion) for every $X \in \mathfrak{X}(M)$.

Let $M$ be a strictly pseudoconvex CR manifold and let $\mathcal{P}_{+}$be the set of all contact forms $\eta$ such that $G_{\eta}$ is positive definite (the set of all positively oriented contact forms on $M$ ). For every $\eta \in \mathcal{P}_{+}$the Webster metric $g_{\eta}$ is a Riemannian metric on $M$. Also there is a constant $C_{n}>0$ (depending only on the CR dimension $n$,cf.e.g. [4]) such that $d \operatorname{vol}\left(g_{\eta}\right)=C_{n} \Psi_{\eta}$ where $d \operatorname{vol}\left(g_{\eta}\right)$ is the canonical volume form of the Riemannian manifold $\left(M, g_{\eta}\right)$. In particular the divergence operators with respect to $d \operatorname{vol}\left(g_{\eta}\right)$ and $\Psi_{\eta}$ coincide.

Let $(S, h)$ be a Riemannian manifold, where $h$ is its metric tensor. Given a relatively compact domain $\Omega \subset \subset M$ let $E_{b, \Omega}: C^{1}(M, S) \rightarrow \mathbb{R}$ be the functional defined by

$$
E_{b, \Omega}(\phi)=\frac{1}{2} \int_{\Omega} \operatorname{trace}_{G_{\eta}}\left(\Pi_{H} \phi^{*} h\right) \Psi_{\eta}
$$

for every $\phi \in C^{1}(M, S)$. If $B$ is a bilinear form on $T(M)$ then $\Pi_{H} B$ denotes its restriction to $H(M) \otimes H(M)$. When $M$ is compact we write simply $E_{b}=E_{b, M}$. A map $\phi \in C^{1}(M, S)$ is a critical point of $E_{b, \Omega}$ if

$$
\frac{d}{d t}\left\{E_{b, \Omega}\left(\phi_{t}\right)\right\}_{t=0}=0
$$

for every smooth 1-parameter variation $\left\{\phi_{t}\right\}_{|t|<\delta} \subset C^{1}(M, S)$ of $\phi$ (i.e. $\phi_{0}=\phi$ ) supported in $\Omega$ i.e. $\operatorname{Supp}(V) \subset \Omega$. Here $V \in C\left(\phi^{-1} T(S)\right)$ is the infinitesimal variation induced by $\left\{\phi_{t}\right\}_{|t|<\delta}$ i.e.

$$
\begin{gathered}
V_{x}=\left(d_{(x, 0)} H\right)(\partial / \partial t)_{(x, 0)}, \quad x \in M, \\
H: M \times(-\delta, \delta) \rightarrow S, \quad H(x, t)=\phi_{t}(x), \quad|t|<\delta .
\end{gathered}
$$

A map $\phi \in C^{1}(M, S)$ is subelliptic harmonic if it is a critical point of $E_{b, \Omega}$ for every domain $\Omega \Subset M$. Let $\left\{X_{a}: 1 \leq a \leq 2 n\right\}$ be a local $G_{\eta}$-orthonormal (i.e. $\left.G_{\eta}\left(X_{a}, X_{b}\right)=\delta_{a b}\right)$ frame of $H(M)$, defined on the domain $U \subset M$ of a local coordinate chart $\chi: U \rightarrow \mathbb{R}^{n+1}$, such that $J X_{\alpha}=X_{\alpha+n}$ for any $1 \leq \alpha \leq n$. Then $\left\{\chi_{*} X_{a}: 1 \leq a \leq 2 n\right\}$ is a Hörmander system on $\chi(U)$ (as a mere consequence of the fact that $T_{1,0}(M)$ is nondegnerate) and, for every subelliptic harmonic map $\phi: M \rightarrow S$, the map $\phi \circ \chi^{-1}: \chi(U) \rightarrow S$ is subelliptic harmonic in the sense of [23]. one sets

Let $\nabla^{h}$ be the Levi-Civita connection of $(S, h)$. Given a $C^{2}$ map $\phi: M \rightarrow S$

$$
\beta_{b}(\phi)(X, Y)=\left(\phi^{-1} \nabla^{h}\right)_{X} \phi_{*} Y-\phi_{*} \nabla_{X} Y, \quad X, Y \in \mathfrak{X}(M),
$$

(a pseudohermitian analog to the second fundamental form of $\phi$ ). Here $\phi^{-1} \nabla^{h}$ is the pullback of $\nabla^{h}$ by $\phi$ (a connection in the pullback bundle $\phi^{-1} T(S) \rightarrow M$ ). 
From now on we assume, for the sake of simplicity, that $M$ is compact. The EulerLagrange equations of the variational principle $\delta E_{b}(\phi)=0$ are $\tau_{b}(\phi)=0$ where $\tau_{b}(\phi) \in C\left(\phi^{-1} T(S)\right)$ is given by

$$
\tau_{b}(\phi)=\operatorname{trace}_{G_{\eta}}\left(\Pi_{H} \beta_{b}(\phi)\right)
$$

and may be locally represented as

$$
\tau_{b}(\phi)^{\alpha}=-\Delta_{b} \phi^{\alpha}+\sum_{a=1}^{2 n}\left(\Gamma_{\beta \gamma}^{\alpha} \circ \phi\right) X_{a}\left(\phi^{\beta}\right) X_{a}\left(\phi^{\gamma}\right)
$$

for any $1 \leq \alpha \leq \operatorname{dim}(S)$. Here $\Gamma_{\beta \gamma}^{\alpha}$ are the Christoffel symbols of $h_{\alpha \beta}=h\left(\partial_{\alpha}, \partial_{\beta}\right)$ relative to the local coordinate system $\left(y^{\alpha}\right)$ on $S$, with $\partial_{\alpha} \equiv \partial / \partial y^{\alpha}$. Also $\Delta_{b} \equiv$ $\Delta_{b, \eta}$ is the sublaplacian of $(M, \eta)$ i.e. the formally self-adjoint, positive, second order differential operator given by

$$
\Delta_{b} u=-\operatorname{div}\left(\nabla^{H} u\right), \quad u \in C^{2}(M)
$$

For every $u \in C^{1}(M)$ the horizontal gradient $\nabla^{H} u \in C(H(M))$ is $\nabla^{H} u=\Pi_{H} \nabla u$, where $\nabla u$ is the ordinary gradient of $u$ with respect to the Webster metric i.e. $g_{\eta}(\nabla u, X)=X(u), X \in \mathfrak{X}(M)$, and $\Pi_{H}: T(M) \rightarrow H(M)$ is the projection associated to the decomposition (2.1). Equations $\tau_{b}(\phi)^{\alpha}=0$ are quasilinear subelliptic hence every $C^{2}$ (actually $C^{0}$ suffices, $c f$. C-J. Xu and C. Zuily [35]) subelliptic harmonic map is $C^{\infty}$.

Let $U \subset M$ be an open subset. A function $u: U \rightarrow \mathbb{R}$ is weakly differentiable on $U$ along the Levi distribution $H(M)$ if there is $Y_{u} \in L_{\text {loc }}^{1}(U, H(M))$ such that

$$
\int_{U} G_{\eta}\left(Y_{u}, X\right) \Psi_{\eta}=-\int_{U} u \operatorname{div}(X) \Psi_{\eta}, \quad X \in C_{0}^{\infty}(U, H(M)) .
$$

Such $Y_{u}$ is uniquely determined, up to a set of measure zero, and denoted by $Y_{u}=\nabla^{H} u$ (the weak horizontal gradient of $u$ ). One thinks of $\nabla^{H}$ as an operator of Hilbert spaces $\nabla^{H}: \mathcal{D}\left(\nabla^{H}\right) \subset L^{2}(U) \rightarrow L^{2}(U, H(M))$ with domain $\mathcal{D}\left(\nabla^{H}\right)=\mathfrak{M}^{1}(U)$ consisting of all weakly differentiable $u \in L^{2}(U)$ such that $\nabla^{H} u \in L^{2}(U, H(M))$. Clearly $C_{0}^{\infty}(U) \subset \mathfrak{M}^{1}(U)$ so that $\nabla^{H}$ is densely defined. Let $\mathfrak{M}^{1}\left(U, \mathbb{R}^{v}\right)$ consist of all maps $\phi=\left(\phi^{1}, \ldots, \phi^{v}\right): U \rightarrow \mathbb{R}^{v}$ such that $\phi^{P} \in \mathfrak{M}^{1}(U)$ for every $1 \leq P \leq v$. If $j: S \hookrightarrow \mathbb{R}^{v}$ is a given isometric immersion, for some $v>\operatorname{dim}(S)$, then $\mathfrak{M}^{1}(U, S)$ consists of all $\phi \in \mathfrak{M}^{1}\left(U, \mathbb{R}^{v}\right)$ such that $\phi(x) \in S$ for a.e. $x \in U$ (and clearly $\mathfrak{M}^{1}(U, S)$ depends on the immersion $j$ ).

For every $u \in \mathfrak{M}^{1}(M)$ and $X \in C^{\infty}(H(M))$ we set by definition $X(u)=$ $G_{\eta}\left(X, \nabla^{H} u\right)$.

Lemma 2.1. If $u, v \in \mathfrak{M}^{1}(M)$ then $u v$ is weakly differentiable along $H(M)$ and $\nabla^{H}(u v)=v \nabla^{H} u+u \nabla^{H} v$. 
Proof. Clearly $v \nabla^{H} u+u \nabla^{H} u \in L_{\text {loc }}^{1}(H(M))$. Let $\left\{u_{\nu}\right\}_{\nu \geq 1}$ and $\left\{v_{\nu}\right\}_{\nu \geq 1}$ such that $u_{v}, v_{v} \in C^{\infty}(M)$ and $u_{v} \rightarrow u, v_{v} \rightarrow v$ in $\mathfrak{M}^{1}(M)$ as $v \rightarrow \infty$. Then for every $X \in C^{\infty}(H(M))$

$$
\begin{aligned}
& \int_{M} G_{\eta}\left(v \nabla^{H} u+u \nabla^{H} u, X\right) \Psi_{\eta} \\
& =\lim _{\nu \rightarrow \infty} \int_{M} G_{\eta}\left(\nabla^{H} u, v_{v} X\right) \Psi_{\eta}+\lim _{\nu \rightarrow \infty} \int_{M} G_{\eta}\left(\nabla^{H} v, u_{v} X\right) \Psi_{\eta} \\
& =-\lim _{\nu \rightarrow \infty}\left\{\int_{M} u \operatorname{div}\left(v_{v} X\right) \Psi_{\eta}+\int_{M} v \operatorname{div}\left(u_{v} X\right) \Psi_{\eta}\right\} \\
& =-\lim _{\nu \rightarrow \infty} \int_{M}\left\{u\left[X\left(v_{\nu}\right)+v_{\nu} \operatorname{div}(X)\right]+v\left[X\left(u_{v}\right)+u_{v} \operatorname{div}(X)\right]\right\} \Psi_{\eta} \\
& =-\int_{M}\{u X(v)+u v \operatorname{div}(X)+v X(u)+v u \operatorname{div}(X)\} \Psi_{\eta} \\
& =-\int_{M}\left\{G_{\eta}\left(u \nabla^{H} v+v \nabla^{H} u, X\right)+2 u v \operatorname{div}(X)\right\} \Psi_{\eta} .
\end{aligned}
$$

The tangential Cauchy-Riemann operator is the first-order differential operator $\bar{\partial}_{b}$ given by $\left(\bar{\partial}_{b} u\right) \bar{Z}=\bar{Z}(u)$ for any $u \in C^{1}(U, \mathbb{C})$ and $Z \in T_{1,0}(M)$. A $C^{1}$ function $u$ is a $C R$ function if $\bar{\partial}_{b} u=0$ (the tangential Cauchy-Riemann equations). A function $u \in L_{\text {loc }}^{1}(U)$ is weakly $C R$ if

$$
\int_{U} u \operatorname{div}(\bar{Z}) \Psi_{\eta}=0, \quad Z \in C_{0}^{\infty}\left(U, T_{1,0}(M)\right) .
$$

Given two CR manifolds $M$ and $S$ a $C R$ map is a $C^{1}$ map $\phi: M \rightarrow N$ such that $\left(d_{x} \phi\right) T_{1,0}(M)_{x} \subset T_{1,0}(S)_{\phi(x)}$ for any $x \in M$. If $S \subset \mathbb{C}^{N}$ is a real hypersurface (thought of as a CR manifold with the induced CR structure $T_{1,0}(S)=[T(S) \otimes$ $\left.\mathbb{C}] \cap T^{1,0}\left(\mathbb{C}^{N}\right)\right)$ then a $C^{1}$ map $\phi=\left(\phi^{1}, \ldots, \phi^{N}\right): M \rightarrow S$ is CR if and only if each $\phi^{A}$ is a CR function on $M$. Also $\phi=\left(\phi^{1}, \ldots, \phi^{N}\right)$ is weakly $C R$ if each $\phi^{A}$ is a weakly CR function.

Let $U \subset M$ be an open set. A function $u \in L_{\text {loc }}^{1}(U)$ has vanishing weak normal derivatives if

$$
\int_{U} u N(\psi) \Psi_{\eta}=0, \quad \psi \in C_{0}^{\infty}(U) .
$$

Here $N=-J T$ and $T \in \mathfrak{X}\left(S^{2 n-1}\right)$ is the Reeb vector field of $(M, \eta)$.

A map $\phi \in \mathfrak{M}^{1}(M, S)$ is weakly subelliptic harmonic, as a map of the pseudohermitian manifold $(M, \eta)$ into the Riemannian manifold $(S, h)$, if for any point $x \in M$ there is an open neighborhood $U \subset M$ carrying a local $G_{\eta}$-orthonormal frame $\left\{T_{\alpha}: 1 \leq \alpha \leq n\right\} \subset C^{\infty}\left(U, T_{1,0}(M)\right)$ such that

$$
\int_{U}\left\{G_{\eta}\left(\nabla^{H} \phi^{P}, \nabla^{H} \psi\right)-2 \psi \sum_{\alpha=1}^{n}\left(\Gamma_{Q R}^{P} \circ \phi\right) T_{\alpha}\left(\phi^{Q}\right) T_{\bar{\alpha}}\left(\phi^{R}\right)\right\} \Psi_{\eta}=0
$$


for every $\psi \in C_{0}^{\infty}(U)$. Existence and regularity of weakly subelliptic harmonic maps were recently studied by L. Capogna and N. Garofalo [8], Z-H. Zhou [37] (for maps from domains in $\mathbb{R}^{m}$ endowed with Hörmander systems of vector fields) and by C. Wang [31], for maps from Carnot groups.

\section{Bergman-harmonic maps}

Let $\Omega=\left\{z \in \mathbb{C}^{n}: \rho(z)<0\right\}$ be a smoothly bounded strictly pseudoconvex domain and let $K_{\Omega}(z, \zeta)$ be its Bergman kernel. Let $g_{\Omega}$ be the Bergman metric on $\Omega$ i.e.

$$
\left(g_{\Omega}\right)_{j \bar{k}}=\frac{\partial^{2} \log K_{\Omega}(z, z)}{\partial z_{j} \partial \bar{z}_{k}}, \quad 1 \leq j, k \leq n .
$$

Let $D \subset \mathbb{C}^{N}$ be a domain endowed with an arbitrary Riemannian metric $h$. Given a domain $A$ such that $\bar{A} \subset \Omega$, the Dirichlet energy functional is

$$
E_{A}(\Phi)=\frac{1}{2} \int_{A}\|d \Phi\|^{2} d \operatorname{vol}\left(g_{\Omega}\right)
$$

for every $\Phi \in C^{2}(\Omega, D)$. Here $\|d \Phi\|: \Omega \rightarrow[0,+\infty)$ is the Hilbert-Schmidt norm of $d \Phi$ i.e. $\|d \Phi\|^{2}=\operatorname{trace}_{g_{\Omega}}\left(\Phi^{*} h\right)$. A $C^{2}$ map $\Phi: \Omega \rightarrow D$ is Bergman-harmonic if

$$
\frac{d}{d t}\left\{E_{A}\left(\Phi_{t}\right)\right\}_{t=0}=0
$$

for any $A \Subset \Omega$ and any smooth 1-parameter variation $\left\{\Phi_{t}\right\}_{|t|<\delta} \subset C^{2}(\Omega, D)$ of $\Phi$ (i.e. $\Phi_{0}=\Phi$ ) supported in $A$ i.e. $\operatorname{Supp}(V) \subset A$ where $V \in C^{1}\left(\Phi^{-1} T(\Omega)\right)$ is the infinitesimal variation induced by $\Phi$

$$
\begin{gathered}
V_{z}=\left(d_{(z, 0)} F\right)(\partial / \partial t)_{(z, 0)}, \quad z \in \Omega, \\
F: \Omega \times(-\delta, \delta) \rightarrow D, \quad F(z, t)=\Phi_{t}(z), \quad|t|<\delta .
\end{gathered}
$$

The first variation formula for the Dirichlet energy is

$$
\frac{d}{d t}\left\{E_{\Omega}\left(\Phi_{t}\right)\right\}_{t=0}=-\int_{\Omega} h^{\Phi}\left(V, \tau_{g_{\Omega}}(\Phi)\right) d \operatorname{vol}\left(g_{\Omega}\right)
$$

where $\tau_{g_{\Omega}}(\Phi) \in C\left(\Phi^{-1} T(D)\right)$ is the tension field of $\Phi$ i.e. $\tau_{g_{\Omega}}(\Phi)=$ trace $_{g_{\Omega}} \beta_{g_{\Omega}}(\Phi)$ and $\beta_{g_{\Omega}}(\Phi)$ is the second fundamental form of $\Phi$

$$
\beta_{g_{\Omega}}(\Phi)(X, Y)=\left(\nabla^{h}\right)_{X}^{\Phi} \Phi_{*} Y-\Phi_{*} \nabla_{X}^{g_{\Omega}} Y
$$

for any $X, Y \in \mathfrak{X}(\Omega)$. Therefore the Euler-Lagrange equations of the variational principle associated to $E_{\Omega}$ are $\tau_{g_{\Omega}}(\Phi)=0$ and these may be written locally

$$
-\Delta_{g_{\Omega}} \Phi^{P}+\left(\Gamma_{Q R}^{P} \circ \Phi\right) \frac{\partial \Phi^{Q}}{\partial x^{a}} \frac{\partial \Phi^{R}}{\partial x^{b}} G^{a b}=0, \quad 1 \leq P \leq 2 N,
$$


where $\Phi^{P}=X^{P} \circ \Phi, \Delta_{g}$ is the Bergman Laplacian, i.e. the Laplace-Beltrami operator of $\left(\Omega, g_{\Omega}\right)$ as a Riemanian manifold, and $\Gamma_{Q R}^{P}$ are the Christoffel symbols of $h_{P Q}=h\left(\partial_{P}, \partial_{Q}\right)$ with $\partial_{P} \equiv \partial / \partial X^{P}$ and $1 \leq P, Q \leq 2 N$. If $\left(z^{1}, \ldots, z^{n}\right)$ and $\left(Z^{1}, \ldots, Z^{N}\right)$ are the Cartesian complex coordinates on $\mathbb{C}^{n}$ and $\mathbb{C}^{N}$ then $z^{j}=$ $x^{j}+i x^{n+j}$ and $Z^{A}=X^{A}+i X^{N+A}$ with $1 \leq j \leq n$ and $1 \leq A \leq N$. We set as customary $G_{a b}=g_{\Omega}\left(\partial_{a}, \partial_{b}\right)$ with $\partial_{a}=\partial / \partial x^{a}$ and $1 \leq a, b \leq 2 n$. Then $\left[G^{a b}\right]=\left[G_{a b}\right]^{-1}$. The Bergman Laplacian is given by

$$
\Delta_{g_{\Omega}} u=-\sum_{a=1}^{2 n}\left\{E_{a}\left(E_{a}(u)\right)-\left(\nabla_{E_{a}}^{g_{\Omega}} E_{a}\right)(u)\right\}, \quad u \in C^{2}(\Omega),
$$

where $\nabla^{g_{\Omega}}$ is the Levi-Civita connection of $\left(\Omega, g_{\Omega}\right)$ and $\left\{E_{a}: 1 \leq a \leq 2 n\right\}$ is a $g_{\Omega}$-orthonormal frame of $T(\Omega)$.

Any \pm holomorphic (i.e. holomorphic or anti-holomorphic) map $\Phi: \mathbb{B}_{n} \rightarrow$ $\mathbb{B}_{N}$ is Bergman-harmonic, with respect to an arbitrary Kählerian metric $h$ on $\mathbb{B}_{N}$. The classical theorem in [28] (that \pm holomorphic maps of compact Kählerian manifolds minimize the Dirichlet energy within their homotopy classes, $c f$. [28]) fails to apply, due to lack of compactness of $\mathbb{B}_{n}$. One may but partially recover Lichnerowicz's result by integrating over a domain $\Omega$ such that $\bar{\Omega} \subset \mathbb{B}_{n}$. Our finding is Theorem 1.1 in Section 1 i.e.

$$
E_{\Omega}(\Psi) \geq\left|E_{\Omega}(\Phi) \pm \int_{\partial \Omega} i_{\partial \Omega}^{*}\left(\alpha(H) \wedge \mathcal{W}^{\mathbb{B}_{n}}\right)\right|
$$

whenever $\Phi$ is \pm holomorphic and a smooth homotopy $H: \Phi \simeq \Psi$ is given. Here $\alpha(H)$ is a differential 1-form determined by $H$ (and discovered by A. Lichnerowicz, cf. op. cit.) and $\mathcal{W}^{\mathbb{B}_{n}}=-i \partial \bar{\partial} \log K_{\mathbb{B}_{n}}(z, z)$ (the Kähler 2-form of $\mathbb{B}_{n}$ ). When the domain is $\Omega=\Omega_{\epsilon}=\{\varphi<-\epsilon\} \subset \mathbb{B}_{n}$ we may estimate the boundary integral $\int_{M_{\epsilon}} u \Psi_{\theta}$ in inequality (3.3) (where $u=\alpha(H)(T) \in C\left(\overline{\mathbb{B}}_{n}\right)$ and $\Psi_{\theta}=\theta \wedge(d \theta)^{n-1}$ ) to show that $E_{\Omega_{\epsilon}}(\Psi) \geq E_{\Omega_{\epsilon}}(\Phi)+O\left(\epsilon^{-n+1}\right)$ as $\epsilon \rightarrow 0$. Our calculations rely on the differential geometric machinery outlined in Section 4 and Section 5 (and due to [19] and [14]).

\section{The method of A. Korányi and H. M. Reimann}

By a celebrated result of C. Fefferman ( $c f$. [17]) one has the asymptotic expansion formula

$$
K_{\Omega}(z, \zeta)=C_{\Omega}|\nabla \rho(\zeta)|^{2} \cdot \operatorname{det} L_{\rho}(\zeta) \cdot \Psi(z, \zeta)^{-(n+1)}+E(z, \zeta)
$$

with $E \in C^{\infty}(\bar{\Omega} \times \bar{\Omega} \backslash \Delta)$ and

$$
|E(z, \zeta)| \leq C_{\Omega}^{\prime}|\Psi(z, \zeta)|^{-(n+1)+1 / 2}|\log | \Psi(z, \zeta)|| .
$$


Here $L_{\rho}$ is the Levi form and $\Delta=\{(z, z): z \in \partial \Omega\}$. Also

$$
\begin{aligned}
& \Psi(z, \zeta)=(F(z, \zeta)-\rho(\zeta)) \chi(|z-\zeta|)+(1-\chi(|z-\zeta|))|z-\zeta|^{2} \\
& F(z, \zeta)=-\sum_{j=1}^{n} \frac{\partial \rho}{\partial z^{j}}(\zeta)\left(z^{j}-\zeta^{j}\right)-\frac{1}{2} \sum_{j, k=1}^{n} \frac{\partial^{2} \rho}{\partial z^{j} \partial z^{k}}(\zeta)\left(z^{j}-\zeta^{j}\right)\left(z^{k}-\zeta^{k}\right)
\end{aligned}
$$

and $\chi(t)$ is a $C^{\infty}$ cut-off function with $\chi(t)=1$ for $|t|<\epsilon_{0} / 2$ and $\chi(t)=0$ for $|t| \geq 3 \epsilon_{0} / 4$. As an elementary consequence of (4.1)-(4.2) if

$$
\varphi(z)=-K_{\Omega}(z, z)^{-1 /(n+1)}
$$

then $\varphi(z) \rightarrow 0$ and $\nabla \varphi(z) \neq 0$ as $z \rightarrow \partial \Omega$ hence $\varphi$ is a defining function for $\Omega$. The observation is essentially due to A. Korányi and H.M. Reimann (cf. [25]) although P. Klembeck was the first ( $c f$. [24]) to exploit (4.1)-(4.2) towards geometric applications. A parallel among the work in [24] and the approach by E. Barletta (cf. [2]) to Klembeck's result will be drawn later on in this paper, as a means to understand the peculiarities of the asymptotic analysis used. As a qualitative consequence of A. Korányi and H.M. Reimann's observation one may effectively relate the Kählerian geometry of the interior of $\Omega$ (springing from its Bergman metric $g_{\Omega}$ ) to the contact geometry of the boundary $\partial \Omega$ (associated to the contact form $\left.\theta=\frac{i}{2}(\bar{\partial}-\partial) \varphi(z)\right)$. Neither the Bergman metric $g_{\Omega}$, nor geometric objects associated to it (the Levi-Civita connection $\nabla^{g} \Omega$, its curvatures, etc.) stay bounded at the boundary (for instance $g_{\Omega}$ is $O\left(\varphi^{-1}\right)$ as $\varphi \rightarrow 0$ ). The quantitatively precise solution is to evaluate $g_{\Omega}$ and $\nabla^{g_{\Omega}}$ on each leaf of the foliation $\mathcal{F}$ by level sets $M_{\epsilon}=\{\varphi=-\epsilon\}, 0<\epsilon \leq \epsilon_{0}$, and approach the boundary as $\epsilon \rightarrow 0$ (i.e. as $\left.M_{\epsilon} \rightarrow \partial \Omega\right) c f$. work by C. R. Graham and J. M. Lee [19]. This is a rather old technique, going back to G. Cimmino ( $c f . e . g$. [9] and [26]) and the actual contribution of C. R. Graham and J. M. Lee is to compute $\nabla^{g_{\Omega}}$ in terms of quantities surviving at the boundary, such as a metric connection (devised by them and referred to by us as the Graham-Lee connection, $c f$. also [14]) whose pointwise restriction to each leaf $M_{\epsilon}$ is the Tanaka-Webster connection of the leaf, and in terms of derivatives of the transverse curvature of $\mathcal{F}$. As an application of the methods in [25] and [19] we study the boundary behavior of Bergman-harmonic maps $\Phi: \mathbb{B}_{n} \rightarrow \mathbb{B}_{N}$. We distinguish two cases, as I) $\lim _{\mathbb{B}_{n} \ni z \rightarrow z_{0} \in S^{2 n-1}} \Phi(z) \in \mathbb{B}_{N}$ and $\mathbb{B}_{N}$ is endowed with a Riemannian metric $h$ such that the pullback $\Phi^{-1} \nabla^{h}$ of the Levi-Civita connection $\nabla^{h}$ stays bounded at the boundary of $\mathbb{B}_{n}$, or II) $\Phi$ is a proper map extending smoothly at the boundary and $h=g_{\mathbb{B}_{N}}$ (the Bergman metric). In the first case we show that the boundary values $\phi: S^{2 n-1} \rightarrow \mathbb{B}_{N}$ of $\Phi$ is a subelliptic harmonic map (in the sense of J. Jost and C-J. Xu [23]) of the pseudohermitian manifold $\left(S^{2 n-1}, \theta\right)$ into the Riemannian manifold $\left(\mathbb{B}_{N}, h\right)$, provided $\phi$ has vanishing normal derivatives. In the second case the boundary values map $\phi: S^{2 n-1} \rightarrow S^{2 N-1}$ is shown to be a solution to the PDEs system $\phi_{\bar{A}} \bar{\partial}_{b} \phi^{A}=0$ where $\bar{\partial}_{b}$ is the tangential Cauchy-Riemann operator on $S^{2 n-1}$ ( $c f$. Theorem 1.2 in Section 1). As a but necessary condition for Bergman-harmonicity, equations (1.5) have of course a 
limited practical use (e.g. may be used to show that the modified Faran maps (5.1) and (5.11) in Section 5 are not Bergman-harmonic).

\section{Smooth maps between balls}

A map $\Phi: \mathbb{B}_{n} \rightarrow \mathbb{B}_{N}$ is proper if $\Phi^{-1}(K)$ is compact in $\mathbb{B}_{n}$ for any compact subset $K \subset \mathbb{B}_{N}$. For maps extending continuously to the boundary, properness is equivalent to $\Phi\left(S^{2 n-1}\right) \subset S^{2 N-1}$. The main examples we keep in mind are maps $\Phi=\left(\Phi^{1}, \ldots, \Phi^{N}\right)$ whose components $\Phi^{A}$ are polynomials, often monomials $c f$. [11]-[13], such that $\Phi\left(S^{2 n-1}\right) \subset S^{2 N-1}$. Two maps $\Phi$ and $\Psi$ are spherically equivalent if $\Phi=\zeta \circ \Psi \circ \xi^{-1}$ for some automorphisms $\xi \in \operatorname{Hol}\left(\mathbb{B}_{n}\right)$ and $\zeta \in \operatorname{Hol}\left(\mathbb{B}_{N}\right)$. Due to the complicated structure of the Bergman-harmonic map system (here with $h=g_{\mathbb{B}_{N}}$ )

$$
\begin{aligned}
& -2\left(1-|z|^{2}\right) \sum_{j, k=1}^{n}\left(\delta_{j k}-z_{j} \bar{z}_{k}\right) \frac{\partial^{2} \Phi^{A}}{\partial z_{j} \partial \bar{z}_{k}} \\
& +\sum_{j=1}^{n} \frac{1}{1-|\Phi|^{2}}\left(\delta_{B}^{A} \bar{\Phi}_{C}+\delta_{C}^{A} \bar{\Phi}_{B}\right) E_{j}\left(\Phi^{B}\right) \bar{E}_{j}\left(\Phi^{C}\right)=0
\end{aligned}
$$

the analysis of particular examples appears as rather involved, too. For instance, the following modification

$$
\Phi(z, w)=\left(|z|^{2} z, \sqrt{3} z w,|w|^{2} w\right), \quad(z, w) \in \mathbb{B}_{2},
$$

of Faran's map $(z, w) \mapsto\left(z^{3}, \sqrt{3} z w, w^{3}\right)$, is proper but not holomorphic. Is (5.1) at least Bergman-harmonic? As announced in Sections 1 and 2, our approach to the study of Bergman-harmonic maps between strictly pseudoconvex domains, and in particular balls, is twofold i.e. we adapt a Lichnerowicz-type argument ( $c f$. [28]) to the case of open Kählerian manifolds ( $c f$. Theorem 1.1) and we study the boundary behavior of a Bergman-harmonic map ( $c f$. Theorem 1.2).

Theorem 5.1. Let $h=g_{\mathbb{B}_{N}}$. If $\Phi: \mathbb{B}_{n} \rightarrow \mathbb{B}_{N}$ is a Bergman-harmonic map then $U \circ \Phi$ and $\zeta \circ \Phi \circ \xi^{-1}$ are Bergman-harmonic for any $U \in \mathrm{U}(N), \xi \in \operatorname{Hol}\left(\mathbb{B}_{n}\right)$ and $\zeta \in \operatorname{Hol}\left(\mathbb{B}_{N}\right)$.

Proof. As $\operatorname{Hol}\left(\mathbb{B}_{N}\right) \subset \operatorname{Isom}\left(\mathbb{B}_{N}, g_{\mathbb{B}_{N}}\right)$ the Hilbert-Schmidt norm of a $C^{2}$ map $\mathbb{B}_{n} \rightarrow \mathbb{B}_{N}$ is a $\mathrm{U}(N)$-invariant. Let $\Omega \Subset \mathbb{B}_{n}$ be a relatively compact subdomain and let $\left\{\Psi_{t}\right\}_{|t|<\delta}$ be a smooth 1-parameter variation of $\Psi=U \circ \Phi$ supported in $\Omega$ i.e. $\operatorname{Supp}(W) \subset \Omega$ where $W \in C^{1}\left(\Psi^{-1} T\left(\mathbb{B}_{n}\right)\right)$ is the infinitesimal variation induced by $\left\{\Psi_{t}\right\}_{|t|<\delta}$. Then $\Phi_{t}=U^{-1} \circ \Psi_{t}$ is a 1 -parameter variation of $\Phi$ supported in $\Omega$ and $E_{\Omega}\left(\Psi_{t}\right)=E_{\Omega}\left(U \circ \Phi_{t}\right)=E_{\Omega}\left(\Phi_{t}\right)$ hence $\Psi$ is a critical point of $E_{\Omega}$. In general if $\Psi=\zeta \circ \Phi \circ \xi^{-1}$ (including the previous case) then $\|d \Psi\|=\|d \Phi\| \circ \xi^{-1}$ (because of $\zeta^{*} g_{\mathbb{B}_{N}}=g_{\mathbb{B}_{N}}$ ) and $E_{\xi(\Omega)}(\Psi)=E_{\Omega}(\Phi)$ (by a change of variable under the integral sign, as $\xi$ is an isometry of $\left.\left(\mathbb{B}_{n}, g_{\mathbb{B}_{n}}\right)\right)$. 
Let $\operatorname{Hol}_{ \pm}(n, N)$ be the set of all \pm holomorphic maps $\mathbb{B}_{n} \rightarrow \mathbb{B}_{N}$. Let $P(n, N)$ consist of all proper maps $\Phi \in \operatorname{Hol}_{+}(n, N)$ extending holomorphically past the boundary of $\mathbb{B}_{n}$. Let $P^{*}(n, N)$ be the quotient of $P(n, N)$ by the relation of spherical equivalence. By a result of J. J. Faran $\left(c f\right.$. [16]) $\sharp\left[P^{*}(2,3)\right]=4$.

Corollary 5.2. The Dirichlet energy $E_{\mathbb{B}_{2}}: P(2,3) \rightarrow \mathbb{R}$ is discrete and the corresponding energy levels are precisely the spherical equivalence classes in $P^{*}(2,3)$.

Let $\Phi: \mathbb{B}_{n} \rightarrow \mathbb{B}_{N}$ be a $C^{\infty}$ map. Let $d^{c} \Phi: T\left(\mathbb{B}_{n}\right) \otimes \mathbb{C} \rightarrow T\left(\mathbb{B}_{N}\right) \otimes \mathbb{C}$ be the $\mathbb{C}$-linear extension of the differential of $\Phi$. We set as customary

$$
\begin{aligned}
& \partial \Phi: T^{1,0}\left(\mathbb{B}_{n}\right) \rightarrow T^{1,0}\left(\mathbb{B}_{N}\right), \quad \partial \Phi=\pi_{1,0} \circ\left(d^{c} \Phi\right) \circ i_{1,0}, \\
& \bar{\partial} \Phi: T^{0,1}\left(\mathbb{B}_{n}\right) \rightarrow T^{1,0}\left(\mathbb{B}_{N}\right), \quad \bar{\partial} \Phi=\pi_{1,0} \circ\left(d^{c} \Phi\right) \circ i_{0,1},
\end{aligned}
$$

where $\pi_{1,0}: T\left(\mathbb{B}_{N}\right) \otimes \mathbb{C} \rightarrow T^{1,0}\left(\mathbb{B}_{N}\right)$ is the projection while $i_{1,0}: T^{1,0}\left(\mathbb{B}_{n}\right) \rightarrow$ $T\left(\mathbb{B}_{n}\right) \otimes \mathbb{C}$ and $i_{0,1}: T^{0,1}\left(\mathbb{B}_{n}\right) \rightarrow T\left(\mathbb{B}_{n}\right) \otimes \mathbb{C}$ are inclusions. Then

$$
\begin{aligned}
& \|\partial \Phi\|^{2}=\left(g_{\mathbb{B}_{n}}\right)^{j \bar{k}} \Phi_{j}^{A} \Phi_{\bar{k}}^{\bar{B}}\left(g_{\mathbb{B}_{N}}\right)_{A \bar{B}}, \quad \Phi_{\bar{j}}^{\bar{A}}=\overline{\Phi_{j}^{A}}, \\
& \|\bar{\partial} \Phi\|^{2}=\left(g_{\mathbb{B}_{n}}\right)^{j \bar{k}} \Phi_{\bar{k}}^{A} \Phi_{j}^{\bar{B}}\left(g_{\mathbb{B}_{N}}\right)_{A \bar{B}}, \quad \Phi_{j}^{\bar{A}}=\overline{\Phi_{\bar{j}}^{A}},
\end{aligned}
$$

where $\Phi_{j}^{A}, \Phi_{j}^{A} \in C^{\infty}\left(\mathbb{B}_{n}, \mathbb{C}\right)$ are given by

$$
(\partial \Phi) \partial / \partial z^{j}=\Phi_{j}^{A} \partial / \partial Z^{A}, \quad(\bar{\partial} \Phi) \partial / \partial \bar{z}^{j}=\Phi_{j}^{A} \partial / \partial Z^{A} .
$$

It follows that

$$
\begin{gathered}
E_{\Omega}(\Phi)=E_{\Omega}^{\prime}(\Phi)+E_{\Omega}^{\prime \prime}(\Phi), \\
E_{\Omega}^{\prime}(\Phi)=\int_{\Omega}\|\partial \Phi\|^{2} d \operatorname{vol}\left(g_{\mathbb{B}_{n}}\right), \quad E_{\Omega}^{\prime \prime}(\Phi)=\int_{\Omega}\|\bar{\partial} \Phi\|^{2} d \operatorname{vol}\left(g_{\mathbb{B}_{n}}\right),
\end{gathered}
$$

for every domain $\Omega \subset \mathbb{C}^{n}$ such that $\bar{\Omega} \subset \mathbb{B}_{n}$. The following is immediate:

Lemma 5.3. A $C^{\infty}$ map $\Phi: \mathbb{B}_{n} \rightarrow \mathbb{B}_{N}$ is \pm holomorphic if and only if $E_{\Omega}^{\prime \prime}(\Phi)=0$ or $E_{\Omega}^{\prime}(\Phi)=0$ for every $\Omega \Subset \mathbb{B}_{n}$.

Let us set $K_{\Omega}(\Phi)=\int_{\Omega}\left(\|\partial \Phi\|^{2}-\|\bar{\partial} \Phi\|^{2}\right) d \operatorname{vol}\left(g_{\mathbb{B}_{n}}\right)$. If $\mathcal{W}^{\mathbb{B}_{n}}$ and $\mathcal{W}^{\mathbb{B}_{N}}$ are the Kähler 2-forms of the Kählerian manifolds $\left(\mathbb{B}_{n}, g_{\mathbb{B}_{n}}\right)$ and $\left(\mathbb{B}_{N}, g_{\mathbb{B}_{N}}\right)$ then let $\left\langle\mathcal{W}^{\mathbb{B}_{n}}, \Phi^{*} \mathcal{W}^{\mathbb{B}_{N}}\right\rangle: \mathbb{B}_{n} \rightarrow \mathbb{R}$ be defined by

$$
\left\langle\mathcal{W}^{\mathbb{B}_{n}}, \Phi^{*} \mathcal{W}^{\mathbb{B}_{N}}\right\rangle=\left(g_{\mathbb{B}_{n}}\right)^{j \bar{k}}\left(g_{\mathbb{B}_{n}}\right)^{\bar{r} s} \mathcal{W}^{\mathbb{B}_{n}}\left(E_{j}, E_{\bar{r}}\right)\left(\Phi^{*} \mathcal{W}^{\mathbb{B}_{N}}\right)\left(E_{\bar{k}}, E_{s}\right) .
$$

One easily checks that

$$
\|\partial \Phi\|^{2}-\|\bar{\partial} \Phi\|^{2}=\left\langle\mathcal{W}^{\mathbb{B}_{n}}, \Phi^{*} \mathcal{W}^{\mathbb{B}_{N}}\right\rangle .
$$


Let $\left\{H_{t}: 0 \leq t \leq 1\right\} \subset C^{\infty}\left(\mathbb{B}_{n}, \mathbb{B}_{N}\right)$ be a smooth 1-parameter family of maps. As $d \mathcal{W}^{\mathbb{B}_{N}}=0$

$$
\left.\frac{\partial}{\partial t}\left(H_{t}^{*} \mathcal{W}^{\mathbb{B}_{N}}\right)=H_{t}^{*} d\left(\frac{\partial H_{t}}{\partial t}\right\lrcorner \mathcal{W}^{\mathbb{B}_{N}}\right) .
$$

Moreover we set

$$
\left.\alpha(H)=\int_{0}^{1} H_{t}^{*}\left\{\frac{\partial H_{t}}{\partial t}\right\lrcorner \mathcal{W}^{\mathbb{B}_{N}}\right\} d t .
$$

If $D \subset \mathbb{C}^{N}$ is convex then any two maps $\Phi, \Psi: \Omega \rightarrow D$ are homotopic.

Proof of Theorem 1.1. (i) By integrating in (5.3)

$$
H_{1}^{*} \mathcal{W}^{\mathbb{B}_{N}}-H_{0}^{*} \mathcal{W}^{\mathbb{B}_{N}}=d \alpha(H)
$$

Consequently (by (5.2) and (5.4))

$$
\begin{aligned}
& K_{\Omega}\left(H_{1}\right)-K_{\Omega}\left(H_{0}\right) \\
& =\int_{\Omega}\left\{\left\langle\mathcal{W}^{\mathbb{B}_{n}}, H_{1}^{*} \mathcal{W}^{\mathbb{B}_{N}}\right\rangle-\left\langle\mathcal{W}^{\mathbb{B}_{n}}, H_{0}^{*} \mathcal{W}^{\mathbb{B}_{N}}\right\rangle\right\} d \operatorname{vol}\left(g_{\mathbb{B}_{n}}\right) \\
& =\int_{\Omega} \mathcal{W}^{\mathbb{B}_{n}} \wedge *\left(H_{1}^{*} \mathcal{W}^{\mathbb{B}_{N}}-H_{0}^{*} \mathcal{W}^{\mathbb{B}_{N}}\right)=\int_{\Omega} \mathcal{W}^{\mathbb{B}_{n}} \wedge * d \alpha(H) \\
& =\int_{\Omega}(d \alpha(H)) \wedge * \mathcal{W}^{\mathbb{B}_{n}}=\int_{\Omega} d\left(\alpha(H) \wedge * \mathcal{W}^{\mathbb{B}_{n}}\right)
\end{aligned}
$$

i.e. (by Stokes' theorem)

$$
K_{\Omega}\left(H_{1}\right)-K_{\Omega}\left(H_{0}\right)=\int_{\partial \Omega} \alpha(H) \wedge * \mathcal{W}^{\mathbb{B}_{n}}
$$

because

$$
* \mathcal{W}^{\mathbb{B}_{n}}=\frac{1}{(n-1) !}\left(\mathcal{W}^{\mathbb{B}_{n}}\right)^{n-1}
$$

yields $d\left(* \mathcal{W}^{\mathbb{B}_{n}}\right)=0$. Finally (by $\left.(5.5)\right)$

$$
\begin{aligned}
E_{\Omega}(\Psi) & =E_{\Omega}^{\prime}(\Psi)+E_{\Omega}^{\prime \prime}(\Psi) \geq\left|E_{\Omega}^{\prime}(\Psi)-E_{\Omega}^{\prime \prime}(\Psi)\right| \\
& =\left|K_{\Omega}(\Psi)\right|=\left|K_{\Omega}(\Phi)+a_{M}(H)\right|= \\
& = \begin{cases}\left|E_{\Omega}^{\prime}(\Phi)+a_{M}(H)\right| & \text { if } \Phi \text { is }+ \text { holomorphic } \\
\left|E_{\Omega}^{\prime \prime}(\Phi)-a_{M}(H)\right| & \text { if } \Phi \text { is }- \text { holomorphic }\end{cases} \\
& =\left|E_{\Omega}(\Phi) \pm a_{M}(H)\right| .
\end{aligned}
$$

(ii) Let $\tilde{H}_{t}=H_{1-t}$ for every $0 \leq t \leq 1$. Then (by a change of variable) $\alpha(\tilde{H})=$ $-\alpha(H)$ yielding (by (1.2)) $E_{\Omega}(\Phi) \geq\left|E_{\Omega}(\Psi) \mp a_{M}(H)\right|$ for every $\Omega \Subset \mathbb{B}_{n}$. If there exist homotopies $H: \Phi \simeq \Psi$ and $H^{\prime}: \Phi \simeq \Psi$ such that $a_{M}(H)=0$ and 
$a_{M}\left(H^{\prime}\right) \neq 0$ then $E_{\Omega}(\Phi)=E_{\Omega}(\Psi)$ and, say for $\Phi$ and $\Psi$ both holomorphic and $a_{M}\left(H^{\prime}\right)>0, E_{\Omega}(\Psi) \geq E_{\Omega}(\Phi)+a_{M}\left(H^{\prime}\right)$, a contradiction.

(iii) We set $\Omega_{\epsilon}=\left\{z \in \mathbb{B}_{n}: \varphi(z)<-\epsilon\right\}$ for every $0<\epsilon \leq \epsilon_{0}$. Here $\varphi(z)=$ $a_{n}\left(|z|^{2}-1\right)$ and we rely on notations and conventions detailed in Section 4 and Section 6. Let $M_{\epsilon}=\partial \Omega_{\epsilon}$. Let $\left\{\omega^{j}: 1 \leq j \leq n\right\}$ be complex valued local $(1,0)$ forms dual to the complex vector fields (6.16) i.e. $\omega^{j}\left(E_{k}\right)=\delta_{k}^{j}$ and $\omega^{j}\left(E_{\bar{k}}\right)=0$. If $\left\{\theta^{\alpha}: 1 \leq \alpha \leq n-1\right\}$ is an adapted coframe of $T_{1,0}(\mathcal{F})$ i.e. $\theta^{\alpha}\left(W_{\beta}\right)=\delta_{\beta}^{\alpha}$, $\theta^{\alpha}\left(W_{\bar{\beta}}\right)=0$ and $\theta^{\alpha}(T)=0$ then

$$
\omega^{\alpha}=\sqrt{-\frac{n+1}{\varphi}} \theta^{\alpha}, \quad \omega^{n}=\sqrt{\frac{n+1}{2 f \varphi}} \partial \varphi
$$

Consequently the Kähler 2-form of $\left(\mathbb{B}_{n}, g_{\mathbb{B}_{n}}\right)$ is given by $(c f .(6.3)-(6.5))$

$$
\mathcal{W}^{\mathbb{B}_{n}}=-2 i \sum_{j=1}^{n} \omega^{j} \wedge \omega^{\bar{j}}=\frac{2 i(n+1)}{\varphi}\left\{\sum_{\alpha} \theta^{\alpha} \wedge \theta^{\bar{\alpha}}-\frac{1-r \varphi}{2 \varphi} \partial \varphi \wedge \bar{\partial} \varphi\right\} .
$$

Then (as $\left.i_{\epsilon}^{*} \partial \varphi=0\right)$

$$
i_{\epsilon}^{*} \mathcal{W}^{\mathbb{B}_{N}}=2 i(n+1) \epsilon^{-1} \sum_{\alpha} \theta^{\alpha} \wedge \theta^{\bar{\alpha}}
$$

Moreover

$$
\begin{aligned}
\left(\sum_{\alpha} \theta^{\alpha} \wedge \theta^{\bar{\alpha}}\right)^{n-1} & =i^{(n-1)(n-2)} \delta_{\alpha_{1} \beta_{1}} \ldots \delta_{\alpha_{n-1} \beta_{n-1}} \theta^{\alpha_{1} \ldots \alpha_{n-1}} \wedge \theta^{\bar{\beta}_{1} \ldots \bar{\beta}_{n-1}}, \\
\theta^{\alpha_{1} \ldots \alpha_{n-1}} & =\theta^{\alpha_{1}} \wedge \ldots \wedge \theta^{\alpha_{n-1}} .
\end{aligned}
$$

Therefore (by (5.6)-(5.7))

$$
i_{\epsilon}^{*}\left(* \mathcal{W}^{\mathbb{B}_{n}}\right)=\epsilon^{-(n-1)} C_{n} i^{(n-1)^{2}} \theta^{1 \ldots(n-1)} \wedge \theta^{\overline{1} \ldots \overline{(n-1)}}
$$

with $C_{n}=(2 n+2)^{n+1} /(n-1)$ !

Lemma 5.4. $u=\alpha(H) T$ extends continuously to the boundary of $\mathbb{B}_{n}$.

Proof. The real 1-form $\alpha(H)$ is given by

$$
\alpha(H)_{z} X=\int_{0}^{1} \mathcal{W}_{H_{t}(z)}^{\mathbb{B}_{n}}\left(\left(d_{(z, t)} H\right)(\partial / \partial t)_{(z, t)},\left(d_{z} H_{t}\right) X\right) d t
$$

for every $X \in T_{z}(\Omega)$ and $z \in \Omega$. Hence (by $T=i z_{j} \partial / \partial z^{j}+$ complex conjugate)

$$
\begin{aligned}
u= & \int_{0}^{1}\left\{\left(\left(g_{\mathbb{B}_{N}}\right)_{A \bar{B}} \circ H\right) \frac{\partial H^{A}}{\partial t}\left(z^{j} \frac{\partial H_{\bar{B}}}{\partial z^{j}}-\bar{z}_{j} \frac{\partial H_{\bar{B}}}{\partial \bar{z}_{j}}\right)\right. \\
& \left.+\left(\left(g_{\mathbb{B}_{N}}\right)_{\bar{A} B} \circ H\right) \frac{\partial H_{\bar{A}}}{\partial t}\left(z^{j} \frac{\partial H^{B}}{\partial z^{j}}-\bar{z}_{j} \frac{\partial H^{B}}{\partial \bar{z}_{j}}\right)\right\} d t
\end{aligned}
$$

hence $u(z)$ stays finite as $\mathbb{B}_{n} \ni z \rightarrow S^{2 n-1}$. 
Finally (by (5.8))

$$
a_{M_{\epsilon}}(H)=C_{n} i^{(n-1)^{2}} \epsilon^{-n+1} \int_{M_{\epsilon}} u \Psi_{\theta}
$$

yielding the estimate (1.3) with $\|u\|_{L^{\infty}}=\sup _{z \in \overline{\mathbb{B}}_{n}}|u(z)|$ and (by $\Psi_{\theta}=2^{n-1}(n-$ $\left.1) ! d \operatorname{vol}\left(g_{\theta}\right)\right)$ with

$$
\operatorname{Vol}\left(M_{\epsilon}, i_{\epsilon}^{*} \theta\right)=\int_{M_{\epsilon}} \Psi_{\theta}=2^{n-1}(n-1) ! \omega_{n-1}\left(1-\epsilon / a_{n}\right)^{(2 n-1) / 2}=O(1)
$$

where $\omega_{n-1}$ is the "area" of $S^{2 n-1}$.

Theorem 5.5. i) Let $H_{t}(z, w)=\left(t z, \sqrt{1-t^{2}} z^{2}, \sqrt{2-t^{2}} z w, w^{2}\right)$ for every $0 \leq$ $t \leq 1$ and $(z, w) \in \mathbb{B}_{2}$. Then $H: \mathbb{B}_{2} \times[0,1] \rightarrow \mathbb{B}_{4}$ is a homotopy of Faran's maps $j_{0} \circ \Phi=H_{0}$ and $j_{1} \circ \Psi=H_{1}$

$$
\Phi(z, w)=\left(z^{2}, \sqrt{2} z w, w^{2}\right), \quad \Psi(z, w)=\left(z, z w, w^{2}\right),
$$

such that each $H_{t}$ is a proper holomorphic map and

$$
\left(\left(g_{\mathbb{B}_{4}}\right)_{A \bar{B}} \circ H\right) \frac{\partial H^{A}}{\partial t}\left(\bar{z} \frac{\partial H_{\bar{B}}}{\partial \bar{z}}+\bar{w} \frac{\partial H_{\bar{B}}}{\partial \bar{w}}\right)
$$

stays bounded at the boundary of $\mathbb{B}_{2}$. Here $j_{0}, j_{1}: \mathbb{B}_{3} \rightarrow \mathbb{B}_{4}$ are the injections $j_{0}(Z)=(0, Z)$ and $j_{1}(Z)=\left(Z_{1}, 0, Z_{2}, Z_{3}\right), Z \in \mathbb{B}_{3}$. In particular $a_{\partial \Omega}(H)=$ 0 for every domain $\Omega \Subset \mathbb{B}_{2}$.

ii) Let $H_{t}(z, w)=\left(t z, \sqrt{1-t^{2}}|z|^{2}, \sqrt{2-t^{2}} z w,|w|^{2}\right),(z, w) \in \mathbb{B}_{2}$. Then $H: \mathbb{B}_{2} \times[0,1] \rightarrow \mathbb{B}_{4}$ is a smooth homotopy $H: j_{0} \circ \Phi \simeq j_{1} \circ \Psi$ of

$$
\Phi(z, w)=\left(|z|^{2}, \sqrt{2} z w,|w|^{2}\right), \quad \Psi(z, w)=\left(z, z w,|w|^{2}\right),
$$

by proper $C^{\infty}$ non \pm holomorphic maps $H_{t}, 0 \leq t \leq 1$. None of the maps (5.11) is Bergman-harmonic. The functions

$$
\left(\left(g_{\mathbb{B}_{4}}\right)_{A \bar{B}} \circ H\right) \frac{\partial H^{A}}{\partial t}\left(z \frac{\partial H_{\bar{B}}}{\partial z}+w \frac{\partial H_{\bar{B}}}{\partial w}\right),
$$

as well as (5.10) stay bounded at the boundary of $\mathbb{B}_{n}$. The boundary integral in (1.2) vanishes i.e. $a_{\partial \Omega}(H)=0$.

Homotopies $H$ in Theorem 5.5 are examples satisfying the basic assumption in (iii) of Theorem 1.1. 
Proof of Theorem 5.5. One notes that

$$
\begin{aligned}
\frac{\partial H^{A}}{\partial t} H_{\bar{A}} & =t|z|^{2}\left(1-|z|^{2}-|w|^{2}\right), \\
\frac{\partial H^{A}}{\partial t} \frac{\partial H_{\bar{A}}}{\partial \bar{z}} \bar{z} & =t|z|^{2}\left(1-2|z|^{2}-|w|^{2}\right), \quad \frac{\partial H^{A}}{\partial t} \frac{\partial H_{\bar{A}}}{\partial \bar{w}} \bar{w}=-t|z|^{2}|w|^{2}, \\
H^{B} \frac{\partial H_{\bar{B}}}{\partial \bar{z}} \bar{z} & =t^{2}|z|^{2}+2\left(1-t^{2}\right)|z|^{4}+\left(2-t^{2}\right)|z|^{2}|w|^{2}, \\
H^{B} \frac{\partial H_{\bar{B}}}{\partial \bar{w}} \bar{w} & =\left(2-t^{2}\right)|z|^{2}|w|^{2}+2|w|^{4}, \\
\left(g_{\mathbb{B}_{N}}\right)_{A \bar{B}} \circ H & =\frac{N+1}{1-|H|^{2}}\left\{\delta_{A B}+\frac{H_{\bar{A}} H^{B}}{1-|H|^{2}}\right\},
\end{aligned}
$$

(with $N=4$ ) hence

$$
\left(\left(g_{\mathbb{B}_{4}}\right)_{A \bar{B}} \circ H\right) \frac{\partial H^{A}}{\partial t}\left(\bar{z} \frac{\partial H_{\bar{B}}}{\partial \bar{z}}+\bar{w} \frac{\partial H_{\bar{B}}}{\partial \bar{w}}\right)=\frac{t|z|^{2}\left(|z|^{2}+|w|^{2}-1\right)^{2}}{\left(1-|H|^{2}\right)^{2}}
$$

tending to $\left(2-t^{2}\left|z_{0}\right|^{2}\right)^{-2}$ as $\mathbb{B}_{2} \ni(z, w) \rightarrow\left(z_{0}, w_{0}\right) \in S^{2 n-1}$. Moreover (as (5.12) is real) $\alpha(H)=0$ and (by (ii) in Theorem 1.1) $E_{A}(\Psi)=E_{A}(\Phi)$ (also $a_{M}\left(H^{\prime}\right)=0$ for any other smooth homotopy $\left.H^{\prime}: \Phi \simeq \Psi\right)$. The Proof of (ii) in Theorem 5.5 is similar and omitted.

\section{Boundary values of Bergman-harmonic maps}

From now on we shall assume that $\Phi: \Omega \rightarrow D$ is a map extending smoothly up to the boundary of $\Omega$ and analyze (3.1) in the limit as $z \rightarrow \partial \Omega$. The first step is to relate $\nabla^{g \Omega}$ to the Graham-Lee connection, a linear connection defined on a one-sided neighborhood of $\partial \Omega$ which we proceed to recall $(c f .[14,19])$.

Let $M_{\epsilon}=\{z \in \Omega: \varphi(z)=-\epsilon\}$ with $\epsilon>0$ be the level hypersurfaces of $\varphi$ (given by (4.3)). There is $\epsilon_{0}>0$ such that $M_{\epsilon}$ is a smooth strictly pseudoconvex CR manifold, of CR dimension $n-1$, for every $0<\epsilon \leq \epsilon_{0}$. Therefore there is a one-sided neighborhood of $V \subset \bar{\Omega}$ of the boundary, carrying a foliation $\mathcal{F}$ whose leaf space is $V / \mathcal{F}=\left\{M_{\epsilon}: 0<\epsilon \leq \epsilon_{0}\right\}$. Let $H(\mathcal{F}) \rightarrow V$ and $T_{1,0}(\mathcal{F}) \rightarrow$ $V$ be respectively the bundles whose portions over a leaf $L \in V / \mathcal{F}$ are the Levi distribution and the CR structure of $L . C f$. [15] for the main notions and conventions in CR and pseudohermitian geometry. Then

$$
\begin{aligned}
& T_{1,0}(\mathcal{F}) \cap T_{0,1}(\mathcal{F})=\{0\}, \quad T_{0,1}(\mathcal{F}) \equiv \overline{T_{1,0}(\mathcal{F})}, \\
& Z, W \in C^{\infty}\left(T_{1,0}(\mathcal{F})\right) \Longrightarrow[Z, W] \in C^{\infty}\left(T_{1,0}(\mathcal{F})\right), \\
& H(\mathcal{F})=\operatorname{Re}\left\{T_{1,0}(\mathcal{F}) \oplus T_{0,1}(\mathcal{F})\right\}
\end{aligned}
$$


By a result of J.M. Lee and R. Melrose [27], there is a unique complex vector field $\xi$ of type $(1,0)$ on $V$ such that

$$
\partial \varphi(\xi)=1, \quad \partial \bar{\partial} \varphi(\xi, \bar{Z})=0, \quad Z \in T_{1,0}(\mathcal{F}) .
$$

The transverse curvature of $\mathcal{F}$ is $r=2 \partial \bar{\partial} \varphi(\xi, \bar{\xi})$. Again by a result in [27] the transverse curvature is smooth up to the boundary i.e. $r \in C^{\infty}(\bar{\Omega})$. Let us set $N=\xi+\bar{\xi}$ and $T=i(\xi-\bar{\xi})$. Then $T(\varphi)=0$ i.e. $T \in T(\mathcal{F})$. Note that

$$
T(\mathcal{F})=H(\mathcal{F}) \oplus \mathbb{R} T .
$$

Let us set $\theta=\frac{i}{2}(\bar{\partial}-\partial) \varphi$ so that the pullback of $\theta$ to each leaf $L \in V / \mathcal{F}$ is a contact form on $L$. We shall need the tensor field $g_{\theta}$ given by

$$
g_{\theta}(X, Y)=(d \theta)(X, J Y), \quad g_{\theta}(X, T)=0, \quad g_{\theta}(T, T)=1,
$$

for any $X, Y \in H(\mathcal{F})$. Here $J$ denotes the complex structure of $\mathbb{C}^{n}$. Up to a sign change the contact forms induced by $\theta$ on the leaves of $\mathcal{F}$ are positively oriented hence $g_{\theta}$ is a Riemannian metric on the bundle $T(\mathcal{F})$ (a tangential Riemannian metric for $\mathcal{F}$ ). The pullback of $g_{\theta}$ to a leaf of $\mathcal{F}$ is the Webster metric of that leaf. Let us also set

$$
L_{\theta}(Z, \bar{W})=-i(d \theta)(Z, \bar{W}), \quad Z, W \in T_{1,0}(\mathcal{F}) .
$$

The Graham-Lee connection of $(V, \mathcal{F})$ is the unique linear connection $\nabla$ on $V$ obeying to the axioms i) $T_{1,0}(\mathcal{F})$ is parallel with respect to $\nabla$, ii) $\nabla L_{\theta}=0, \nabla T=$ $0, \nabla N=0$ and iii) the torsion tensor field $T_{\nabla}$ of $\nabla$ is pure i.e.

$$
\begin{gathered}
T_{\nabla}(Z, W)=0, \quad T_{\nabla}(Z, \bar{W})=2 i L_{\theta}(Z, \bar{W}) T, \\
T_{\nabla}(N, W)=r W+i \tau(W), \\
\tau\left(T_{1,0}(\mathcal{F})\right) \subset T_{0,1}(\mathcal{F}), \quad \tau(N)=-J \nabla^{H} r-2 r T,
\end{gathered}
$$

for any $Z, W \in T_{1,0}(\mathcal{F})$ where $\tau(X)=T_{\nabla}(T, X)$ for every $X \in T(V)$. Here $\nabla^{H} r$ (the horizontal gradient of $r$ ) is given by

$$
\nabla^{H} r=\Pi_{H} \nabla r, \quad g_{\theta}(\nabla r, X)=X(r), \quad X \in T(\mathcal{F}),
$$

and $\Pi_{H}: T(\mathcal{F}) \rightarrow H(\mathcal{F})$ is the projection associated to the direct sum decomposition (6.1). Mere differentiation of $\varphi(z)=-K(z, z)^{-1 /(n+1)}$ yields

$$
g_{\Omega}(X, Y)=\frac{n+1}{\varphi}\left\{\frac{i}{\varphi}(\partial \varphi \wedge \bar{\partial} \varphi)(X, J Y)-(d \theta)(X, J Y)\right\}
$$

for any $X, Y \in T(V)$. The identity (6.2) together with the decompositions (6.1) and $T(V)=T(\mathcal{F}) \oplus \mathbb{R} N$ leads to

$$
\begin{aligned}
& g_{\Omega}(X, Y)=-\frac{n+1}{\varphi} g_{\theta}(X, Y), \quad X, Y \in H(\mathcal{F}), \\
& g_{\Omega}(X, T)=0, \quad g_{\Omega}(X, N)=0, \quad X \in H(\mathcal{F}), \\
& g_{\Omega}(T, N)=0, \quad g_{\Omega}(T, T)=g_{\Omega}(N, N)=\frac{n+1}{\varphi f},
\end{aligned}
$$


where $f \equiv \varphi /(1-r \varphi)$. Then

$$
\nabla^{g} u=\frac{\varphi}{n+1}\left\{-\nabla^{H} u+f[T(u) T+N(u) N]\right\}
$$

for every $u \in C^{1}(\Omega)$. As a consequence of equations (6.3)-(6.5) the connections $\nabla^{g} \Omega$ and $\nabla$ are related by

$$
\begin{aligned}
\nabla_{X}^{g_{\Omega}} Y= & \nabla_{X} Y+\left\{f g_{\theta}(\tau X, Y)+g_{\theta}\left(X, J_{b} Y\right)\right\} T-\left\{g_{\theta}(X, Y)\right. \\
& \left.+f g_{\theta}\left(X, J_{b} \tau Y\right)\right\} N \\
\nabla_{X}^{g_{\Omega}} T= & \tau X-\frac{1}{f} J_{b} X-\frac{f}{2}\left\{X(r) T+\left(J_{b} X\right)(r) N\right\} \\
\nabla_{X}^{g_{\Omega}} N= & -\frac{1}{f} X+\tau J_{b} X+\frac{f}{2}\left\{\left(J_{b} X\right)(r) T-X(r) N\right\}, \\
\nabla_{T}^{g_{\Omega}} X= & \nabla_{T} X-\frac{1}{f} J_{b} X-\frac{f}{2}\left\{X(r) T+\left(J_{b} X\right)(r) N\right\}, \\
\nabla_{N}^{g_{\Omega}} X= & \nabla_{N} X-\frac{1}{\varphi} X+\frac{f}{2}\left\{\left(J_{b} X\right)(r) T-X(r) N\right\} \\
\nabla_{N}^{g_{\Omega}} T= & -\frac{1}{2} J_{b} \nabla^{H} r-\frac{f}{2}\left\{\left(N(r)+\frac{4}{\varphi^{2}}-\frac{2 r}{\varphi}\right) T+T(r) N\right\}, \\
\nabla_{T}^{g_{\Omega}} N= & \frac{1}{2} J_{b} \nabla^{H} r-\frac{f}{2}\left\{\left(N(r)+\frac{4}{\varphi^{2}}-\frac{6 r}{\varphi}+4 r^{2}\right) T+T(r) N\right\}, \\
\nabla_{T}^{g_{\Omega}} T= & -\frac{1}{2} \nabla^{H} r-\frac{f}{2}\left\{T(r) T-\left(N(r)+\frac{4}{\varphi^{2}}-\frac{6 r}{\varphi}+4 r^{2}\right) N\right\}, \\
\nabla_{N}^{g_{\Omega}} N=- & \frac{1}{2} \nabla^{H} r+\frac{f}{2}\left\{T(r) T-\left(N(r)+\frac{4}{\varphi^{2}}-\frac{2 r}{\varphi}\right) N\right\},
\end{aligned}
$$

for any $X, Y \in H(\mathcal{F})$. Here $J_{b}: T(\mathcal{F}) \rightarrow T(\mathcal{F})$ is the bundle morphism defined by $J_{b} X=J X$ for every $X \in H\left(\mathcal{F}\right.$ ) and $J_{b} T=0$ (so that $J_{b}^{2}=-I+\theta \otimes T$ on $T(\mathcal{F})$ ). At this point we may prove Theorem 1.2. Let $\left\{W_{\alpha}: 1 \leq \alpha \leq n-1\right\}$ be a local orthonormal (i.e. $\left.g_{\theta}\left(W_{\alpha}, W_{\bar{\beta}}\right)=\delta_{\alpha \beta}\right)$ frame of $T_{1,0}(\mathcal{F})$ and let us set

$$
E_{\alpha}=\sqrt{-\frac{\varphi}{n+1}} W_{\alpha}, \quad E_{n}=\sqrt{\frac{2 f \varphi}{n+1}} \xi
$$

so that (by (6.3)-(6.5)) $\left\{E_{j}: 1 \leq j \leq n\right\}$ is a local orthonormal frame of $T^{1,0}(V)$ (the holomorphic tangent bundle over $V$ ). Then the Bergman Laplacian (3.2) may 
be written as

$$
\begin{aligned}
\Delta_{g_{\Omega}} u= & -\sum_{j=1}^{n}\left\{E_{j} E_{\bar{j}} u+E_{\bar{j}} E_{j} u-\left(\nabla_{E_{j}}^{g_{\Omega}} E_{\bar{j}}\right) u-\left(\nabla_{E_{\bar{j}}}^{g_{\Omega}} E_{j}\right) u\right\} \\
= & \frac{\varphi}{n+1} \sum_{\alpha=1}^{n-1}\left\{W_{\alpha} W_{\bar{\alpha}} u+W_{\bar{\alpha}} W_{\alpha} u-\left(\nabla_{W_{\alpha}}^{g_{\Omega}} W_{\bar{\alpha}}\right) u-\left(\nabla_{W_{\bar{\alpha}}}^{g_{\Omega}} W_{\alpha}\right) u\right\} \\
& -\frac{2 f \varphi}{n+1}\left\{\xi \bar{\xi} u+\bar{\xi} \xi u-\left(\nabla_{\xi}^{g_{\Omega}} \bar{\xi}\right) u-\left(\nabla_{\bar{\xi}}^{g_{\Omega}} \xi\right) u\right\}
\end{aligned}
$$

or, by (6.7)-(6.15),

$$
\Delta_{g_{\Omega}}=-\frac{\varphi}{n+1} \Delta_{b}+\frac{2 \varphi(n-1)}{n+1} N-\frac{f \varphi}{n+1}\left\{N^{2}+T^{2}+\nabla^{H} r+2 r N\right\}
$$

where $\Delta_{b}$ is given by

$$
\Delta_{b} u=-\sum_{\alpha=1}^{n-1}\left(W_{\alpha} W_{\bar{\alpha}}+W_{\bar{\alpha}} W_{\alpha}-\nabla_{W_{\alpha}} W_{\bar{\alpha}}-\nabla_{W_{\bar{\alpha}}} W_{\alpha}\right) u
$$

For any $z \in V$ the definition of $\left(\Delta_{b} u\right)(z)$ does not depend upon the choice of local orthonormal frame $\left\{W_{\alpha}: 1 \leq \alpha \leq n-1\right\}$ of $T_{1,0}(\mathcal{F})$ at $z$. Also $\Delta_{b}$ restricts to each leaf of $\mathcal{F}$ as the sublaplacian of the leaf. If $\left[\left(g_{\Omega}\right)^{j \bar{k}}\right]=\left[\left(g_{\Omega}\right)_{j \bar{k}}\right]^{-1}$ then

$$
\left[G^{a b}\right]=\left(\begin{array}{c}
\frac{1}{4}\left[\left(g_{\Omega}\right)^{j \bar{k}}+\left(g_{\Omega}\right)^{\bar{j} k}\right]-\frac{1}{4 i}\left[\left(g_{\Omega}\right)^{j \bar{k}}-\left(g_{\Omega}\right)^{\bar{j} k}\right] \\
\frac{1}{4 i}\left[\left(g_{\Omega}\right)^{j \bar{k}}-\left(g_{\Omega}\right)^{\bar{j} k}\right] \frac{1}{4}\left[\left(g_{\Omega}\right)^{j \bar{k}}+\left(g_{\Omega}\right)^{\bar{j} k}\right]
\end{array}\right)
$$

so that

$$
\begin{aligned}
\left(\Gamma_{Q R}^{P} \circ \Phi\right) \frac{\partial \Phi^{Q}}{\partial x^{a}} \frac{\partial \Phi^{R}}{\partial x^{b}} G^{a b} & =2\left(\Gamma_{Q R}^{P} \circ \Phi\right) \frac{\partial \Phi^{Q}}{\partial z^{j}} \frac{\partial \Phi^{R}}{\partial \bar{z}^{k}}\left(g_{\Omega}\right)^{j \bar{k}} \\
& =2 \sum_{j=1}^{n}\left(\Gamma_{Q R}^{P} \circ \Phi\right) E_{j}\left(\Phi^{Q}\right) \bar{E}_{j}\left(\Phi^{R}\right)
\end{aligned}
$$

Proof of Theorem 1.2. The Bergman-harmonic map system (3.1) may be written (by (6.16))

$$
\begin{aligned}
& -\Delta_{b} \Phi^{P}+2 \sum_{\alpha=1}^{n-1}\left(\Gamma_{Q R}^{P} \circ \Phi\right) W_{\alpha}\left(\Phi^{Q}\right) W_{\bar{\alpha}}\left(\Phi^{R}\right)+2(n-1) N\left(\Phi^{P}\right) \\
& -f\left\{N^{2}+T^{2}+\nabla^{H} r+2 r N\right\}\left(\Phi^{P}\right)-4 f\left(\Gamma_{Q R}^{P} \circ \Phi\right) \xi\left(\Phi^{Q}\right) \bar{\xi}\left(\Phi^{R}\right)=0
\end{aligned}
$$


hence, as $\varphi \rightarrow 0$

$$
-\Delta_{b} \phi^{P}+2 \sum_{\alpha=1}^{n-1}\left(\Gamma_{Q R}^{P} \circ \Phi\right) W_{\alpha}\left(\phi^{Q}\right) W_{\bar{\alpha}}\left(\phi^{R}\right)+2(n-1) N\left(\phi^{P}\right)=0 .
$$

Consequently one may express the normal derivatives of $\phi$ in terms of purely tangential quantities (a typical non-elliptic phenomenon)

$$
N\left(\phi^{P}\right)=-\frac{1}{2(n-1)} \tau_{b}(\phi)^{P}
$$

which is (1.4) in Theorem 1.2. To prove statement (2) one observes that if $F^{A}=$ $\Phi^{A}+i \Phi^{N+A}$ for every $1 \leq A \leq N$ then

$$
\sum_{j=1}^{n}\left(\Gamma_{Q R}^{P} \circ \Phi\right) E_{j}\left(\Phi^{Q}\right) \bar{E}_{j}\left(\Phi^{R}\right)= \begin{cases}\operatorname{Re} \gamma^{A}(\Phi) & \text { if } P=A \\ \operatorname{Im} \gamma^{A}(\Phi) & \text { if } P=N+A,\end{cases}
$$

where $\gamma^{A}(\Phi)=\sum_{j=1}^{n}\left(\gamma_{B C}^{A} \circ \Phi\right) E_{j}\left(F^{B}\right) \bar{E}_{j}\left(F^{C}\right)$ and

$$
\gamma_{B C}^{A}=\left(g_{D}\right)^{A \bar{E}} \partial\left(g_{D}\right)_{B \bar{E}} / \partial Z^{C}
$$

are the complex coefficients of $\nabla^{g} D$ (the Levi-Civita connection of $\left(D, g_{D}\right)$ ). Identity (6.19) is an elementary consequence of

$$
\begin{gathered}
\Gamma_{B C}^{A}=\Gamma_{B, N+C}^{N+A}=-\Gamma_{N+B, N+C}^{A}=(1 / 2)\left(\gamma_{B C}^{A}+\gamma_{\bar{B} \bar{C}}^{\bar{A}}\right), \\
\Gamma_{B C}^{N+A}=\Gamma_{B, N+C}^{A}=-\Gamma_{N+B, N+C}^{N+A}=(1 / 2 i)\left(\gamma_{B C}^{A}-\gamma_{\bar{B} \bar{C}}^{\bar{A}}\right),
\end{gathered}
$$

for any $1 \leq A, B, C \leq N$. Thus (by (6.17)) the Bergman-harmonic map system (3.1) becomes

$$
\begin{aligned}
& -\Delta_{b} F^{A}+2(n-1) N\left(F^{A}\right)-f\left\{N^{2}+T^{2}+\nabla^{H} r+2 r N\right\}\left(F^{A}\right) \\
& -\frac{2(n+1)}{\varphi} \sum_{j=1}^{n}\left(\gamma_{B C}^{A} \circ \Phi\right) E_{j}\left(F^{B}\right) \bar{E}_{j}\left(F^{C}\right)=0 .
\end{aligned}
$$

If $D=\mathbb{B}_{N}$ and $h=g_{D}$ then

$$
\left(g_{\mathbb{B}_{N}}\right)_{A \bar{B}}=\frac{N+1}{1-|Z|^{2}}\left(\delta_{A B}+\frac{\bar{Z}_{A} Z_{B}}{1-|Z|^{2}}\right), \quad\left(g_{\mathbb{B}_{N}}\right)^{A \bar{B}}=\frac{1-|Z|^{2}}{N+1}\left(\delta^{A B}-Z^{A} \bar{Z}^{B}\right),
$$

so that

$$
\gamma_{B C}^{A}=\frac{1}{1-|Z|^{2}}\left(\delta_{B}^{A} \bar{Z}_{C}+\delta_{C}^{A} \bar{Z}_{B}\right) .
$$


Consequently, by (6.16),

$$
\begin{aligned}
& \frac{n+1}{a_{n}} Q_{\Phi} \sum_{j=1}^{n}\left(\gamma_{B C}^{A} \circ \Phi\right) E_{j}\left(F^{B}\right) \bar{E}_{j}\left(F^{C}\right) \\
& =\left(\delta_{B}^{A} F_{\bar{C}}+\delta_{C}^{A} F_{\bar{B}}\right)\left\{\sum_{\alpha=1}^{n-1} W_{\alpha}\left(F^{B}\right) W_{\bar{\alpha}}\left(F^{C}\right)-2 f \xi\left(F^{B}\right) \bar{\xi}\left(F^{C}\right)\right\}
\end{aligned}
$$

where $a_{n} \equiv\left(\pi^{n} / n !\right)^{1 /(n+1)}$. Also if $\Omega=\{\varphi<0\}$ and $D=\{\tilde{\varphi}<0\}$ then for every proper map $\Phi: \Omega \rightarrow D$ one adopts the notation

$$
\begin{aligned}
Q_{\Phi}(z) & =C_{n, N} \frac{\tilde{\varphi}(\Phi(z))}{\varphi(z)}, \quad z \in \Omega, \\
C_{n, N} & \equiv(N !)^{1 /(N+1)}(n !)^{-1 /(n+1)} \pi^{n /(n+1)-N /(N+1)},
\end{aligned}
$$

agreeing with (3.1) in [12, page 85], when $\Omega=\mathbb{B}_{n}$ and $D=\mathbb{B}_{N}$. Let us substitute from (6.21) into (6.20) and take the limit as $\varphi \rightarrow 0$ in the resulting equation. We obtain

$$
\left(\delta_{B}^{A} \phi_{\bar{C}}+\delta_{C}^{A} \phi_{\bar{B}}\right) \sum_{\alpha=1}^{n-1} W_{\alpha}\left(\phi^{B}\right) W_{\bar{\alpha}}\left(\phi^{C}\right)=0
$$

for any $1 \leq A \leq N$. Let us contract with $\phi_{\bar{A}}$ in (6.23). Then (by the constraint $\left.\phi^{B} \Phi_{\bar{B}}=1\right)$

$$
\sum_{\alpha=1}^{n-1}\left|W_{\bar{\alpha}}\left(\phi^{C}\right) \phi_{\bar{C}}\right|^{2}=0
$$

or $W_{\bar{\alpha}}\left(\phi^{C}\right) \phi_{\bar{C}}=0$ thus yielding $\sum_{A=1}^{N} \phi_{\bar{A}} \bar{\partial}_{b} \phi_{A}=0$ which is (1.5) in Theorem 1.2. The "disappearance" of the second order terms in (6.21), leading to the linear first-order system (1.5), may be thought of as a rather curious phenomenon. An inspection of work in [2] provides a heuristic explanation. As recalled in Section 3 , exploiting Fefferman's expansion formula for the Bergman kernel towards geometric applications is already present in the work by P. Klembeck [24], who proved that the holomorphic sectional curvature of $g_{\Omega}$ tends to to the (constant) holomorphic sectional curvature of $g_{\mathbb{B}_{n}}$

$$
k_{g}(\sigma) \rightarrow-\frac{4}{n+1}, \quad z \rightarrow \partial \Omega,
$$

for every holomorphic plane $\sigma \subset T_{z}(\Omega)$ i.e. $\operatorname{dim}_{\mathbb{R}} \sigma=2$ and $J_{z}(\sigma)=\sigma$. P. Klembeck's work is previous to the discovery of the Graham-Lee connection $\nabla$ and relies on a direct asymptotic evaluation of the sectional curvature, resulting into rather involved calculations. Curvature is computed in terms of $g_{j \bar{k}}(z)$ and their derivatives, and an approximate inverse of $\left[g_{j k}(z)\right]$ is devised in the process, as a matrix asymptotically equivalent to $\left[g^{j \bar{k}}(z)\right]$ as $z \rightarrow \partial \Omega$. A simpler differential geometric proof 
of Klembeck's result, making use of (6.7)-(6.15), is due to E. Barletta [2], who proves that

$$
\begin{aligned}
k_{g}(\sigma) & =\frac{g_{z}\left(R_{z}^{g}(X, Y) Y, X\right)}{g_{z}(X, X) g_{z}(Y, Y)-g_{z}(X, Y)^{2}} \\
& =-\frac{\varphi(z)}{n+1}\left\{-4 k_{\theta}(\sigma)+\frac{4}{f(z)}-2 f(z) \frac{A_{z}(X, X)^{2}+A_{z}\left(X, J_{z} X\right)^{2}}{g_{\theta, z}(X, X)^{2}}\right\}
\end{aligned}
$$

where $k_{\theta}$ is a pseudohermitian analog to the notion of holomorphic sectional curvature introduced by S. M. Webster [33]. The latter is devised to be 1 for the sphere, as well as Riemannian sectional curvature, and $a$ priori one expects that $k_{\theta}$ should contribute to get $-4 /(n+1)$ at the boundary. Since $k_{\theta}$ and $A$ (the pseudohermitian sectional curvature and torsion of the leaves of $\mathcal{F}$ ) stay finite at the boundary (and give respectively the pseudohermitian sectional curvature and the pseudohermitian torsion of $\left(\partial \Omega, i_{\partial \Omega}^{*} \theta\right)$ in the limit as $\left.z \rightarrow \partial \Omega\right)$ and $f(z) \rightarrow 0, \varphi(z) / f(z) \rightarrow 1$ as $z \rightarrow \partial \Omega$, it follows that (6.24) holds yet $k_{\theta}(\sigma)$ gives no quantitative contribution to the result. Similarly, in the asymptotic analysis of the Bergman-harmonic map system for $C^{\infty}$ maps $\Phi: \Omega \rightarrow \mathbb{B}_{N}$, elimination of $O\left(\varphi^{-2}\right)$ coefficients followed by approaching the boundary with $\varphi \rightarrow 0$ "kills" second order terms (only terms with $O\left(\varphi^{-2}\right)$ coefficients may "survive" at the boundary) and one is left with equations (1.5).

The Bergman kernel of $\Omega=\mathbb{B}_{n}$ is given by

$$
K_{\mathbb{B}_{n}}(z, \zeta)=\frac{n ! \pi^{-n}}{(1-z \cdot \bar{\zeta})^{n+1}} .
$$

Thus $\varphi(z)=a_{n}\left(|z|^{2}-1\right)$ and $\mathcal{F}$ is the foliation of $\overline{\mathbb{B}}_{n} \backslash\{0\}$ by spheres

$$
\left\{S^{2 n-1}\left(\sqrt{1-\epsilon / a_{n}}\right): 0 \leq \epsilon<a_{n}\right\} .
$$

Moreover $T_{1,0}(\mathcal{F})$ is locally the span of $\left\{T_{\alpha} \equiv \partial / \partial z^{\alpha}-\left(\bar{z}_{\alpha} / \bar{z}_{n}\right) \partial / \partial z^{n}: 1 \leq \alpha \leq\right.$ $n-1\}$ hence

$$
\xi=\left(1 / a_{n}\right)|z|^{-2} z^{j} \partial / \partial z^{j}
$$

and the transverse curvature of $\mathcal{F}$ is $r=\left(1 / a_{n}\right)|z|^{-2}$. In particular $T(r)=0$ and $X(r)=0$ for every $X \in H(\mathcal{F})$ (with the corresponding simplification of (6.7)(6.15)). Compatibility relations (1.5) become

$$
\Phi_{\bar{A}}\left(z^{n} \frac{\partial \Phi^{A}}{\partial \bar{z}^{\alpha}}-z^{\alpha} \frac{\partial \psi^{A}}{\partial \bar{z}^{n}}\right)=0 .
$$

If $n=2$ then (6.26) is the single equation $\Phi_{\bar{A}}\left(w \partial \Phi^{A} / \partial \bar{z}-z \partial \Phi^{A} / \partial \bar{w}\right)=0$ (and the proper map (5.1) is not Bergman-harmonic). 


\section{Bergman-harmonic maps with $\mathfrak{M}^{1}$-boundary data}

Let $W^{1}\left(\mathbb{B}_{n}\right)$ be the Sobolev space of all $L^{2}$ functions $u: \mathbb{B}_{n} \rightarrow \mathbb{R}$ possessing weak $L^{2}$ derivatives. Let $W^{1}\left(\mathbb{B}_{n}, \mathbb{B}_{N}\right)$ consist of all maps $\Phi=\left(\Phi^{1}, \ldots, \Phi^{2 N}\right)$ : $\mathbb{B}_{n} \rightarrow \mathbb{B}_{N} \subset \mathbb{R}^{2 N}$ such that $\Phi^{P} \in W^{1}\left(\mathbb{B}_{n}\right)$ for every $1 \leq P \leq 2 N$. A map $\Phi \in W^{1}\left(\mathbb{B}_{n}, \mathbb{B}_{N}\right)$ is weakly Bergman-harmonic if

$$
\int_{\mathbb{B}_{n}}\left\{g\left(\nabla^{g} \Phi^{P}, \nabla^{g} \Psi\right)-\Psi\left(\Gamma_{Q R}^{P} \circ \Phi\right) \frac{\partial \Phi^{Q}}{\partial x^{a}} \frac{\partial \Phi^{R}}{\partial x^{b}} G^{a b}\right\} d \operatorname{vol}(g)=0
$$

for any $\Psi \in C_{0}^{\infty}\left(\mathbb{B}_{n}\right)$, where $g=g_{\mathbb{B}_{n}}$. For ones needs in this section $W^{1}\left(\mathbb{B}_{n}\right)$ consists of all $u \in L^{2}\left(\mathbb{B}_{n}\right)$ admitting a weak gradient $\nabla^{g} u \in L^{2}\left(T\left(\mathbb{B}_{n}\right)\right.$. Hence for each $u \in W^{1}\left(\mathbb{B}_{n}\right)$ there exist functions $\left(\nabla^{g} u\right)^{a} \in L^{2}\left(\mathbb{B}_{n}\right)$ such that $\nabla^{g} u=$ $\left(\nabla^{g} u\right)^{a} \partial / \partial x^{a}$ and we set by definition

$$
\frac{\partial u}{\partial x^{b}}=\left(\nabla^{g} u\right)^{a} G_{a b}
$$

and observe that

$$
\int_{\mathbb{B}_{n}} \frac{\partial u}{\partial x^{b}} \Psi d \operatorname{vol}(g)=\int_{\mathbb{B}_{n}} u\left(\frac{\partial}{\partial x^{b}}\right)^{*} \Psi d \operatorname{vol}(g), \quad \Psi \in C_{0}^{\infty}\left(\mathbb{B}_{n}\right),
$$

where $\left(\partial / \partial x^{a}\right)^{*} h=-\partial h / \partial x^{a}-h \operatorname{div}_{g}\left(\partial / \partial x^{a}\right), h \in C_{0}^{1}\left(\mathbb{B}_{n}\right)$, is the formal adjoint of $\partial / \partial x^{a}$. In particular

$$
G^{a b} \frac{\partial \Phi^{Q}}{\partial x^{a}} \frac{\partial \Phi^{R}}{\partial x^{b}}=g\left(\nabla^{g} \Phi^{Q}, \nabla^{g} \Phi^{R}\right) .
$$

Let $u: \mathbb{B}_{n} \rightarrow \mathbb{R}$ and $0<\lambda<1$. As $\mathbb{B}_{n}$ is $\delta_{\lambda}$-contractible the function $u_{\lambda}$ : $S^{2 n-1} \rightarrow \mathbb{R}, u_{\lambda}(\zeta)=u(\lambda \zeta), \zeta \in S^{2 n-1}$, is well defined. If $u \in L^{2}\left(\mathbb{B}_{n}\right)$ then

$$
\|u\|_{\mathbb{B}_{n}}^{2}=\int_{0}^{1} \lambda^{2 n-1}\left\|u_{\lambda}\right\|_{L^{2}\left(S^{2 n-1}\right)}^{2} d \lambda
$$

Given $\Phi: \mathbb{B}_{n} \rightarrow \mathbb{B}_{N}$ and $0<\lambda<1$ let $\Phi_{\lambda}: S^{2 n-1} \rightarrow \mathbb{B}_{N}, \Phi_{\lambda}(\zeta)=\Phi(\lambda \zeta)$, $\zeta \in S^{2 n-1}$. Following [9] a weakly Bergman-harmonic map $\Phi \in W^{1}\left(\mathbb{B}_{n}, \mathbb{B}_{N}\right)$ is said to have $L^{2}$ boundary values if there is $\phi \in L^{2}\left(S^{2 n-1}, \mathbb{R}^{2 N}\right)$ such that $\Phi_{\lambda} \rightarrow \phi$ in $L^{2}\left(S^{2 n-1}, \mathbb{R}^{2 N}\right)$ as $\lambda \rightarrow 1$. $C f$. also [6] and [26].

Proof of Theorem 1.3. We seek for the compatibility conditions satisfied by $\phi$ on $S^{2 n-1}$. As a consequence of (6.7)-(6.15)

$$
\begin{aligned}
& \operatorname{div}_{g}(X)=\operatorname{trace}\left\{Y \in H(\mathcal{F}) \mapsto \nabla_{Y} X\right\} \\
& \operatorname{div}_{g}(T)=0, \\
& \operatorname{div}_{g}(N)=-\frac{2(n-1)}{f}-\frac{4}{\varphi}
\end{aligned}
$$


for every $C^{1}$ section $X$ in $H(\mathcal{F})$, where $\mathcal{F}$ is the foliation of $\overline{\mathbb{B}}_{n} \backslash\{0\}$ by the spheres (6.25) and $\operatorname{div}_{g}$ is the divergence operator with respect to the volume form $d \operatorname{vol}(g)$. Indeed if $V=\mathbb{B}_{n} \backslash\{0\}$ then

$$
r=\frac{1}{a_{n}+\varphi}, \quad f=\frac{1}{a_{n}}\left(a_{n}+\varphi\right) \varphi, \quad N(r)=-\frac{2}{\left(a_{n}+\varphi\right)^{2}} .
$$

In particular $N(r)+2 r^{2}=0$ and equations (6.7)-(6.15) with $\tau=0$ become

$$
\begin{aligned}
& \nabla_{X}^{g} Y=\nabla_{X} Y+g_{\theta}\left(X, J_{b} Y\right) T-g_{\theta}(X, Y) N, \\
& \nabla_{X}^{g} T=-\frac{1}{f} J_{b} X, \quad \nabla_{X}^{g} N=-\frac{1}{f} X, \\
& \nabla_{T}^{g} X=\nabla_{T} X-\frac{1}{f} J_{b} X, \quad \nabla_{N}^{g} X=\nabla_{N} X-\frac{1}{\varphi} X, \\
& \nabla_{N}^{g} T=-\left(\frac{2}{\varphi}+r\right) T, \quad \nabla_{T}^{g} N=-\left(\frac{2}{\varphi}-r\right) T, \\
& \nabla_{T}^{g} T=\left(\frac{2}{\varphi}-r\right) N, \quad \nabla_{N}^{g} N=-\left(\frac{2}{\varphi}+r\right) N .
\end{aligned}
$$

Consequently

$$
\begin{aligned}
& \nabla_{\xi}^{g} X=\nabla_{\xi} X-\frac{1}{2}\left\{\frac{1}{\varphi} X-\frac{i}{f} J_{b} X\right\}=\nabla_{\xi} X-\frac{1}{2 \varphi}\left\{X-\frac{i a_{n}}{a_{n}+\varphi} J_{b} X\right\}, \\
& \nabla_{\xi}^{g} T=i\left(-\frac{2}{\varphi} \xi+r \bar{\xi}\right), \quad \nabla_{\xi}^{g} N=-\frac{2}{\varphi} \xi-r \bar{\xi} .
\end{aligned}
$$

Let $\left\{W_{\alpha}: 1 \leq \alpha \leq n-1\right\}$ be a local $g_{\theta}$-orthonormal frame of $T_{1,0}(\mathcal{F})$. Then (by (7.6))

$$
\nabla_{W_{\alpha}}^{g} X=\nabla_{W_{\alpha}} X-2 g_{\theta}\left(W_{\alpha}, X\right) \bar{\xi},
$$

so that, by (7.11) and (7.13),

$$
g\left(\nabla_{W_{\alpha}}^{g} X, W_{\bar{\alpha}}\right)=-\frac{n+1}{\varphi} g_{\theta}\left(\nabla_{W_{\alpha}} X, W_{\bar{\alpha}}\right), \quad g\left(\nabla_{\xi}^{g} X, \bar{\xi}\right)=0,
$$

as $H(\mathcal{F})$ is $\nabla$-parallel. Thus

$$
\begin{aligned}
\operatorname{div}_{g}(X)= & \sum_{j=1}^{n}\left\{g\left(\nabla_{E_{j}}^{g} X, E_{\bar{j}}\right)+g\left(\nabla_{E_{\bar{j}}}^{g} X, E_{j}\right)\right\} \\
= & -\frac{\varphi}{n+1} \sum_{\alpha=1}^{n-1}\left\{g\left(\nabla_{W_{\alpha}}^{g} X, W_{\bar{\alpha}}\right)+g\left(\nabla_{W_{\bar{\alpha}}}^{g} X, W_{\alpha}\right)\right\} \\
& +\frac{2 f \varphi}{n+1}\left\{g\left(\nabla_{\xi}^{g} X, \bar{\xi}\right)+g\left(\nabla_{\bar{\xi}}^{g} X, \xi\right)\right\}
\end{aligned}
$$


yielding (7.3). Similarly, by (7.7) and (7.12),

$$
g\left(\nabla_{W_{\alpha}}^{g} T, W_{\bar{\alpha}}\right)=\frac{i(n+1)}{\varphi f}, \quad g\left(\nabla_{\xi}^{g} T, \bar{\xi}\right)=-\frac{i(n+1)}{\varphi^{2} f},
$$

yielding (7.4). Finally, by (7.7) and (7.12),

$$
g\left(\nabla_{W_{\alpha}}^{g} N, W_{\bar{\alpha}}\right)=\frac{n+1}{\varphi f}, \quad g\left(\nabla_{\xi}^{g} N, \bar{\xi}\right)=-\frac{n+1}{\varphi^{2} f},
$$

(as $\left.g(\xi, \xi)=[(n+1) / 2] \varphi^{-1} f^{-1}\right)$ and a calculation similar to the above leads to (7.5). Formulae (7.3)-(7.5) may be used to compute the weak $\nabla^{g}$ gradient of each $u \in W^{1}\left(\mathbb{B}_{n}\right)$

$$
\int_{\mathbb{B}_{n}} g\left(\nabla^{g} u, Y\right) d \operatorname{vol}(g)=-\int_{\mathbb{B}_{n}} u \operatorname{div}_{g}(Y) d \operatorname{vol}(g),
$$

for every $Y \in C_{0}^{\infty}\left(T\left(\mathbb{B}_{n}\right)\right)$. By $T(V)=H(\mathcal{F}) \oplus \mathbb{R} T \oplus \mathbb{R} N$ there exist $a, b \in L^{2}(V)$ and $X_{u} \in L^{2}(H(\mathcal{F}))$ such that $\nabla^{g} u=X_{u}+a T+b N$ hence (by (6.3)-(6.5))

$$
g\left(\nabla^{g} u, T\right)=a \frac{n+1}{\varphi f}
$$

or

$$
\int_{\mathbb{B}_{n}} g\left(\nabla^{g} u, \gamma \varphi f T\right) d \operatorname{vol}(g)=(n+1) \int_{\mathbb{B}_{n}} a \gamma d \operatorname{vol}(g)
$$

for every $\gamma \in C_{0}^{\infty}\left(\mathbb{B}_{n}\right)$. Thus (by (7.14), (7.4) and $T(\varphi)=T(f)=0$ )

$$
\begin{aligned}
\int_{\mathbb{B}_{n}} a \gamma d \operatorname{vol}(g) & =-\frac{1}{n+1} \int_{\mathbb{B}_{n}} u \operatorname{div}_{g}(\gamma \varphi f T) d \operatorname{vol}(g) \\
& =-\frac{1}{n+1} \int_{\mathbb{B}_{n}} u \varphi f T(\gamma) d \operatorname{vol}(g) .
\end{aligned}
$$

As

$$
N(\varphi)=2, \quad N(f)=\frac{2(1+r \varphi)}{1-r \varphi}
$$

one has

$$
N(\gamma \varphi f)-\gamma[2(n-1) \varphi+4 f]=\varphi f N(\gamma)+2 \varphi \gamma(r f-n+1) .
$$

hence, by (7.14) and (7.5),

$$
\begin{aligned}
\int_{\mathbb{B}_{n}} b \gamma d \operatorname{vol}(g) & =-\frac{1}{n+1} \int_{\mathbb{B}_{n}} u \operatorname{div}_{g}(\gamma \varphi f N) d \operatorname{vol}(g) \\
& =-\frac{1}{n+1} \int_{\mathbb{B}_{n}} u \varphi f\left\{N(\gamma \varphi f)+2\left(r-\frac{n-1}{f}\right) \gamma\right\} d \operatorname{vol}(g) .
\end{aligned}
$$


Finally, by (6.3)-(6.5), (7.14) and (7.3),

$$
\begin{aligned}
\int_{\mathbb{B}_{n}} g\left(X_{u}, Y\right) d \operatorname{vol}(g) & =-\int_{\mathbb{B}_{n}} u \operatorname{div}_{g}(Y) d \operatorname{vol}(g) \\
& =-\int_{\mathbb{B}_{n}} u \operatorname{trace}\left\{Z \in H(\mathcal{F}) \mapsto \nabla_{Z}(Y)\right\} d \operatorname{vol}(g)
\end{aligned}
$$

for every $Y \in C_{0}^{\infty}(H(\mathcal{F}))$. Let $\Phi \in W^{1}\left(\mathbb{B}_{n}, \mathbb{B}_{N}\right)$ be a weakly Bergman-harmonic map. Then (by (7.15)-(7.17) with $u=\Phi^{P}$ and (6.6)) for any $\Psi \in C_{0}^{\infty}\left(\mathbb{B}_{n}\right)$

$$
\begin{aligned}
& \int_{\mathbb{B}_{n}} g\left(\nabla^{g} \Phi^{P}, \nabla^{g} \Psi\right) d \operatorname{vol}(g)=-\frac{1}{(n+1)} \int_{\mathbb{B}_{n}} \varphi \Phi^{P} \\
& \quad \times\left\{\Delta_{b} \Psi+f T^{2}(\Psi)+f\left[N^{2}(\Psi)+2\left(r-\frac{n-1}{f}\right) N(\Psi)\right]\right\} d \operatorname{vol}(g) .
\end{aligned}
$$

It is the proper place to describe our technique of "approaching" the boundary of $\mathbb{B}_{n}$ in equations (7.1), with the expectation of obtaining equations (2.2) at the boundary, provided that the boundary datum $\phi$ has vanishing weak normal derivatives. The basic idea in the asymptotic analysis of the pointwise equations (6.18) was to approach the boundary by traveling with the leaves $\varphi=-\epsilon$ as $\epsilon \rightarrow 0^{+}$. The integral counterpart of this technique is of course to write (7.1) in polar coordinates, allowing some freedom in the radial part ( $c f$. our choice of $\lambda$ below). The main technical difficulty, given a test function $\psi$ on $S^{2 n-1}$, is to produce a family of test functions $\left\{\Psi^{\lambda}\right\}_{0 \leq \lambda \leq 1}$ on $\mathbb{B}_{n}$ reducing to $\psi$ in the angular (as opposed to radial) part of the integral (7.1), as $\lambda \rightarrow 1^{-}$. The naive solution is to set $\Psi(z)=\psi(z /|z|)$ for each $z \in \mathbb{B}_{n}$. This is infinite at the origin, so one should cut off a suitable neighborhood of $0 \in \mathbb{B}_{n}$, and its support does not lie entirely in $\mathbb{B}_{n}$ [points in $\left\{\zeta \in S^{2 n-1}: \psi(\zeta) \neq 0\right\}$ belong to the support of $\Psi$ ], so one should cut off a one-sided neighborhood of the boundary, as well. The solution would be to set $\Psi^{\lambda}(z)=\psi(z /|z|) \chi^{\lambda}(|z|)$, where $\chi^{\lambda}$ is the square wave $\chi^{\lambda}(t)=1$ for $\epsilon_{0}<t<\lambda$ and $\chi^{\lambda}(t)=0$ elsewhere. Yet $\Psi^{\lambda}$ isn't smooth, so one should replace $\chi^{\lambda}$ by a $C^{\infty}$ cut-off function supported in a slightly larger interval, containing $\left[\epsilon_{0}, \lambda\right]$.

Let $\psi \in C^{\infty}\left(S^{2 n-1}\right), \epsilon_{0}>0$, and $\epsilon_{0}<\lambda<1$ be arbitrarily fixed. Next let $\left\{\chi_{\nu}^{\lambda}\right\}_{\nu \geq 1} \subset C_{0}^{\infty}(\mathbb{R})$ such that $0 \leq \chi_{\nu}^{\lambda}(t) \leq 1$, and $\chi_{\nu}^{\lambda}(t)=1$ for $\epsilon_{0}<t<\lambda$ and $\chi_{v}^{\lambda}(t)=0$ for $t \leq \epsilon_{0}-1 / v$ and $t \geq \lambda+1 / v$. Moreover we set

$$
\Psi_{v}^{\lambda}(z)= \begin{cases}\psi\left(\frac{z}{|z|}\right) \chi_{v}^{\lambda}(|z|) & \text { for } \epsilon_{0}-\frac{1}{v}<|z|<\lambda+\frac{1}{v} \\ 0 & \text { otherwise }\end{cases}
$$

for every $z \in \mathbb{B}_{n}$. Then $\left\{\Psi_{v}^{\lambda}\right\}_{\nu \geq 1} \subset C_{0}^{\infty}\left(\mathbb{B}_{n}\right)$ hence (by (7.1) with $\Psi=\Psi_{v}^{\lambda}$ )

$$
I_{1}^{P}\left(\Psi_{v}^{\lambda}\right)-I_{2}^{P}\left(\Psi_{v}^{\lambda}\right)=0, \quad 1 \leq P \leq 2 N,
$$


where

$$
\begin{gathered}
I_{1}^{P}(\Psi) \equiv \int_{\mathbb{B}_{n}} g\left(\nabla^{g} \Phi^{P}, \nabla^{g} \Psi\right) d \operatorname{vol}(g), \quad I_{2}^{P}(\Psi) \equiv \int_{\mathbb{B}_{n}} \Psi \Gamma^{P}(\Phi) d \operatorname{vol}(g), \\
\Gamma^{P}(\Phi) \equiv\left(\Gamma_{Q R}^{P} \circ \Phi\right) \frac{\partial \Phi^{Q}}{\partial x^{a}} \frac{\partial \Phi^{R}}{\partial x^{b}} G^{a b}
\end{gathered}
$$

Consider the vector field $W: \mathbb{C}^{n} \backslash\{0\} \rightarrow S^{2 n-1}$ given by $W(z)=z /|z|$ for every $z \in \mathbb{C}^{n} \backslash\{0\}$. Then

$$
\begin{aligned}
\left(\Delta_{b} \Psi_{v}^{\lambda}\right)(z) & =\chi_{v}^{\lambda}(|z|) \Delta_{b}(\psi \circ W)(z), \\
T^{2}\left(\Psi_{v}^{\lambda}\right)_{z} & =\chi_{v}^{\lambda}(|z|) T^{2}(\psi \circ W)_{z}, \\
N\left(\Psi_{v}^{\lambda}\right)_{z} & =\chi_{v}^{\lambda}(|z|) N(\psi \circ W)_{z}+\frac{\left(\chi_{v}^{\lambda}\right)^{\prime}(|z|)}{a_{n}|z|} \psi(W(z)), \\
N^{2}\left(\Psi_{v}^{\lambda}\right)_{z}= & \chi_{v}^{\lambda}(|z|) N^{2}(\psi \circ W)_{z}+2 \frac{\left(\chi_{v}^{\lambda}\right)^{\prime}(|z|)}{a_{n}|z|} N(\psi \circ W)_{z} \\
& +\frac{1}{a_{n}^{2}|z|^{2}}\left\{\left(\chi_{v}^{\lambda}\right)^{\prime \prime}(|z|)-\frac{1}{|z|}\left(\chi_{v}^{\lambda}\right)^{\prime}(|z|)\right\} \psi(W(z)),
\end{aligned}
$$

for every $\epsilon_{0}-1 / \nu<|z|<\lambda+1 / \nu$. Note that

$$
\lim _{\nu \rightarrow \infty} \int_{\epsilon_{0}-1 / \nu}^{\lambda+1 / \nu} \chi_{\nu}^{\lambda}(t) G(t) d t=\int_{\epsilon_{0}}^{\lambda} G(t) d t
$$

for any $G \in L^{2}(0,1)$. Indeed

$$
\begin{aligned}
& \left|\int_{\epsilon_{0}-1 / v}^{\lambda+1 / v} \chi_{v}^{\lambda}(t) G(t) d t-\int_{\epsilon_{0}}^{\lambda} G(t) d t\right| \\
& \leq\left|\int_{\epsilon_{0}-1 / v}^{\epsilon_{0}} \chi_{v}^{\lambda}(t) G(t) d t\right|+\left|\int_{\lambda}^{\lambda+1 / v} \chi_{\nu}^{\lambda}(t) G(t) d t\right|
\end{aligned}
$$

and for instance

$$
\begin{aligned}
\left|\int_{\epsilon_{0}-1 / v}^{\epsilon_{0}} \chi_{v}^{\lambda}(t) G(t) d t\right| & \leq \int_{\epsilon_{0}-1 / v}^{\epsilon_{0}}|G(t)| d t \\
& \leq \frac{1}{\sqrt{v}}\|G\|_{L^{2}(0,1)} \rightarrow 0, \quad v \rightarrow \infty
\end{aligned}
$$

Let $d \sigma_{t}$ be the volume element on $S^{2 n-1}(t)$ for every $0<t \leq 1$ (so that $d \sigma_{1}=$ $\left.d \operatorname{vol}\left(g_{\eta}\right)\right)$. Note that

$$
\varphi(t \zeta)=a_{n}\left(t^{2}-1\right), \quad f(t \zeta)=a_{n} t^{2}\left(t^{2}-1\right)
$$


for any $0<t<1$ and $\zeta \in S^{2 n-1}$. Then

$$
\begin{aligned}
& \int_{\mathbb{B}_{n}} \varphi \Phi^{P}\left(\Delta_{b} \Psi_{v}^{\lambda}\right) d \operatorname{vo}(g)=\int_{0}^{1} d t \int_{|z|=t} \varphi(z) \Phi^{P}(z)\left(\Delta_{b} \Psi_{v}^{\lambda}\right)(z) d \sigma_{t}(z) \\
& =a_{n} \int_{\epsilon_{0}-1 / v}^{\lambda+1 / v} t^{2 n-1}\left(t^{2}-1\right) \chi_{v}^{\lambda}(t) \int_{S^{2 n-1}} \Phi_{t}^{P}(\zeta) \Delta_{b}(\psi \circ W)(t \zeta) d \sigma_{1}(\zeta)
\end{aligned}
$$

so that, by (7.2),

$$
\begin{aligned}
& \lim _{\nu \rightarrow \infty} \int_{\mathbb{B}_{n}} \varphi \Phi^{P}\left(\Delta_{b} \Psi_{\nu}^{\lambda}\right) d \operatorname{vo}(g) \\
& =a_{n} \int_{\epsilon_{0}}^{\lambda} t^{2 n-1}\left(t^{2}-1\right) \int_{S^{2 n-1}} \Phi_{t}^{P}(\zeta) \Delta_{b}(\psi \circ W)(t \zeta) d \sigma_{1}(\zeta)
\end{aligned}
$$

To legitimate applying (7.20) let us set

$$
G(t)=t^{2 n-1}\left(t^{2}-1\right) \int_{S^{2 n-1}} \Phi_{t}^{P} \Delta_{b}(\psi \circ W)_{t} d \sigma_{1}
$$

so that

$$
\begin{aligned}
|G(t)|^{2} & \leq t^{2(2 n-1)}\left(\int_{S^{2 n-1}}\left|\Phi_{t}^{P}\right|\left|\Delta_{b}(\psi \circ W)_{t}\right| d \sigma_{1}\right)^{2} \\
& \leq t^{2(2 n-1)}\left\|\Phi_{t}^{P}\right\|_{L^{2}\left(S^{2 n-1}\right)}^{2}\left\|\Delta_{b}(\psi \circ W)_{t}\right\|_{L^{2}\left(S^{2 n-1}\right)}^{2}
\end{aligned}
$$

and, by (7.33),

$$
t^{2 n-1}\left\|\Delta_{b}(\psi \circ W)_{t}\right\|_{L^{2}\left(S^{2 n-1}\right)}^{2} \leq t^{2 n-5}\left\|\Delta_{b} \psi\right\|_{L^{2}\left(S^{2 n-1}\right)}^{2} .
$$

Finally if $n \geq 3$ then, by (7.2),

$$
\int_{0}^{1}|G(t)|^{2} d t \leq\left\|\Delta_{b} \psi\right\|_{L^{2}\left(S^{2 n-1}\right)}^{2}\left\|\Phi^{P}\right\|_{L^{2}\left(\mathbb{B}_{n}\right)}^{2}<\infty
$$

i.e. $G \in L^{2}(0,1)$. Similar to $(7.21)$

$$
\begin{aligned}
& \lim _{\nu \rightarrow \infty} \int_{\mathbb{B}_{n}} \varphi f \Phi^{P} T^{2}\left(\Psi_{\nu}^{\lambda}\right) d \operatorname{vol}(g) \\
& =a_{n}^{2} \int_{\epsilon_{0}}^{\lambda} t^{2 n+1}\left(t^{2}-1\right)^{2} \int_{S^{2 n-1}} \Phi_{t}^{P}(\zeta) T^{2}(\psi \circ W)(t \zeta) d \sigma_{1}(\zeta) .
\end{aligned}
$$

Moreover

$$
\int_{\mathbb{B}_{n}} \varphi f \Phi^{P}\left\{N^{2}\left(\Psi_{\nu}^{\lambda}\right)+2\left(r-\frac{n-1}{f}\right) N\left(\Psi_{\nu}^{\lambda}\right)\right\} d \operatorname{vol}(g)=\sum_{i=1}^{3} J_{i}^{P}(\nu)
$$


where

$$
\begin{aligned}
J_{1}^{P}(v)= & a_{n} \int_{\epsilon_{0}-1 / v}^{\lambda+1 / v} t^{2 n-1}\left(t^{2}-1\right) \chi_{v}^{\lambda}(t) d t \\
& \times \int_{S^{2 n-1}} \Phi_{t}^{P}(\zeta)\left\{a_{n} t^{2}\left(t^{2}-1\right) N^{2}(\psi \circ W)(t \zeta)\right. \\
& \left.+2\left(t^{2}-n\right) N(\psi \circ W)(t \zeta)\right\} d \sigma(\zeta) \\
J_{2}^{P}(v)= & \int_{\epsilon_{0}-1 / v}^{\lambda+1 / v} t^{2 n-2}\left(t^{2}-1\right)\left(\chi_{v}^{\lambda}\right)^{\prime}(t) d t \\
& \times \int_{S^{2 n-1}} \Phi_{t}^{P}(\zeta)\left\{2 a_{n} t^{2}\left(t^{2}-1\right) N(\psi \circ W)(t \zeta)\right. \\
& \left.+\left(t^{2}-2 n+1\right) \psi(\zeta)\right\} d \sigma_{1}(\zeta), \\
J_{3}^{P}(v)= & \int_{\epsilon_{0}-1 / v}^{\lambda+1 / v} t^{2 n-1}\left(t^{2}-1\right)^{2}\left(\chi_{v}^{\lambda}\right)^{\prime \prime}(t) \int_{S^{2 n-1}} \Phi_{t}^{P}(\zeta) \psi(\zeta) d \sigma_{1}(\zeta) .
\end{aligned}
$$

Clearly, as in (7.21)-(7.22) above,

$$
\begin{aligned}
\lim _{\nu \rightarrow \infty} J_{1}^{P}(\nu)= & a_{n} \int_{\epsilon_{0}}^{\lambda} t^{2 n-1}\left(t^{2}-1\right) d t \\
& \times \int_{S^{2 n-1}} \Phi_{t}^{P}(\zeta)\left\{a_{n} t^{2}\left(t^{2}-1\right) N^{2}(\psi \circ W)(t \zeta)\right. \\
& \left.+2\left(t^{2}-n\right) N(\psi \circ W)(t \zeta)\right\} d \sigma_{1}(\zeta)
\end{aligned}
$$

To compute the remaining $\lim _{v \rightarrow \infty} J_{i}^{P}(v)$ we need the explicit construction of $\chi_{v}^{\lambda}$ i.e.

$$
\begin{aligned}
\chi_{v}^{\lambda}(t) & =h\left(1+v\left(t-\epsilon_{0}\right)\right) h(1+v(\lambda-t)), \\
h(t) & =\frac{g(t)}{g(t)+g(1-t)}, \quad g(t)= \begin{cases}\exp \left(-1 / t^{2}\right) & t>0 \\
0 & t \leq 0 .\end{cases}
\end{aligned}
$$

Then

$$
\lim _{\nu \rightarrow \infty} J_{i}^{P}(v)=0, \quad i \in\{2,3\}
$$

We may conclude that

$$
\begin{aligned}
& \lim _{\nu \rightarrow \infty} \int_{\mathbb{B}_{n}} \varphi f \Phi^{P}\left\{N^{2}\left(\Psi_{\nu}^{\lambda}\right)+2\left(r-\frac{n-1}{f}\right) N\left(\Psi_{\nu}^{\lambda}\right)\right\} d \operatorname{vol}(g) \\
& =a_{n} \int_{\epsilon_{0}}^{\lambda} t^{2 n-1}\left(t^{2}-1\right) d t \int_{S^{2 n-1}} \Phi_{t}^{P}(\zeta)\left\{a_{n} t^{2}\left(t^{2}-1\right) N^{2}(\psi \circ W)(t \zeta)\right. \\
& \left.\quad+2\left(t^{2}-n\right) N(\psi \circ W)(t \zeta)\right\} d \sigma_{1}(\zeta) .
\end{aligned}
$$


By gathering the information in (7.18), (7.21)-(7.22) and (7.24) we get

$$
\begin{aligned}
& \lim _{\nu \rightarrow \infty} I_{1}^{P}\left(\Psi_{v}^{\lambda}\right) \\
&=-\frac{a_{n}}{n+1} \int_{\epsilon_{0}}^{\lambda} t^{2 n-1}\left(t^{2}-1\right) d t \int_{S^{2 n-1}} \Phi_{t}^{P}(\zeta)\left\{\Delta_{b}(\psi \circ W)(t \zeta)\right. \\
&+a_{n} t^{2}\left(t^{2}-1\right)\left[T^{2}(\psi \circ W)(t \zeta)+N^{2}(\psi \circ W)(t \zeta)\right] \\
&\left.+2\left(t^{2}-n\right) N(\psi \circ W)(t \zeta)\right\} d \sigma_{1}(\zeta) .
\end{aligned}
$$

Finally

$$
\lim _{\nu \rightarrow \infty} I_{2}^{P}(\nu)=\int_{\epsilon_{0}}^{\lambda} t^{2 n-1} d t \int_{S^{2 n-1}} \psi(\zeta) \Gamma^{P}(\Phi)(t \zeta) d \sigma_{1}(\zeta)
$$

so that, taking the limit of (7.19) as $v \rightarrow \infty$ and replacing from (7.25)-(7.26),

$$
\begin{aligned}
& \int_{\epsilon_{0}}^{\lambda} t^{2 n-1} d t \int_{S^{2 n-1}}\left\{( t ^ { 2 } - 1 ) \Phi _ { t } ^ { P } ( \zeta ) \left[\Delta_{b}(\psi \circ W)(t \zeta)\right.\right. \\
& \quad+a_{n} t^{2}\left(t^{2}-1\right)\left(T^{2}(\psi \circ W)(t \zeta)+N^{2}(\psi \circ W)(t \zeta)\right) \\
& \left.\left.\quad+2\left(t^{2}-n\right) N(\psi \circ W)(t \zeta)\right]+\frac{n+1}{a_{n}} \psi(\zeta) \Gamma^{P}(\Phi)(t \zeta)\right\} d \sigma_{1}(\zeta)=0
\end{aligned}
$$

for every $\epsilon_{0}<\lambda<1$. The reason one used an arbitrary $\lambda \in\left(\epsilon_{0}, 1\right)$ in the construction of $\chi_{v}^{\lambda}(t)$ becomes apparent now, for the fundamental lemma of calculus applies in the $L^{2}$ context i.e. if $G(\lambda)=\int_{\epsilon_{0}}^{\lambda} g(t) d t$ with $g \in L^{2}(0,1)$ then $G^{\prime}=g$ in distributional sense i.e. $T_{G}^{\prime}=T_{g}$ where $T_{G}(\gamma)=\int_{0}^{1} G(\lambda) \gamma(\lambda) d \lambda$ is the distribution associated to $G$. Indeed if $\left\{g_{\nu}\right\}_{\nu \geq 1} \subset C_{0}^{\infty}(0,1)$ is such that $\lim _{\nu \rightarrow \infty} g_{v}=g$ in $L^{2}(0,1)$ then

$$
\begin{aligned}
T_{G}^{\prime}(\gamma) & =-T_{G}\left(\gamma^{\prime}\right)=-\lim _{\nu \rightarrow \infty} \int_{0}^{1} \gamma^{\prime}(\lambda) d \lambda \int_{\epsilon_{0}}^{\lambda} g_{\nu}(t) d t \\
& =\lim _{\nu \rightarrow \infty} \int_{0}^{1} \gamma(\lambda) g_{\nu}(\lambda) d \lambda=T_{g}(\gamma)
\end{aligned}
$$

for every $\gamma \in C_{0}^{\infty}(0,1)$. Differentiation (in distributional sense) with respect to $\lambda$ in (7.27) gives

$$
\begin{aligned}
& \int_{S^{2 n-1}}\left\{( \lambda ^ { 2 } - 1 ) \Phi _ { \lambda } ^ { P } ( \zeta ) \left[\Delta_{b}(\psi \circ W)(\lambda \zeta)\right.\right. \\
& \quad+a_{n} \lambda^{2}\left(\lambda^{2}-1\right)\left(T^{2}(\psi \circ W)(\lambda \zeta)+N^{2}(\psi \circ W)(\lambda \zeta)\right) \\
& \left.\left.\quad+2\left(\lambda^{2}-n\right) N(\psi \circ W)(\lambda \zeta)\right]+\frac{n+1}{a_{n}} \psi(\zeta) \Gamma^{P}(\Phi)(\lambda \zeta)\right\} d \sigma_{1}(\zeta)=0
\end{aligned}
$$


Moreover

$$
\begin{aligned}
\Gamma^{P}(\Phi)(\lambda \zeta)= & -\frac{2 a_{n}}{n+1}\left(\lambda^{2}-1\right) \sum_{\alpha=1}^{n-1} \Gamma_{Q R}^{P}\left(\Phi_{\lambda}(\zeta)\right) W_{\alpha}\left(\Phi^{Q}\right)_{\lambda \zeta} W_{\bar{\alpha}}\left(\Phi^{R}\right)_{\lambda \zeta} \\
& +\frac{4 a_{n}^{2}}{n+1} \lambda^{2}\left(\lambda^{2}-1\right)^{2} \Gamma_{Q R}^{P}\left(\Phi_{\lambda}(\zeta)\right) \xi\left(\Phi^{Q}\right)_{\lambda \zeta} \bar{\xi}\left(\Phi^{R}\right)_{\lambda \zeta}
\end{aligned}
$$

hence (7.28) simplifies to

$$
\begin{aligned}
& \int_{S^{2 n-1}}\left\{\Phi_{\lambda}^{P}(\zeta) \Delta_{b}(\psi \circ W)(\lambda \zeta)\right. \\
& \left.\quad-2 \psi(\zeta) \sum_{\alpha=1}^{n-1} \Gamma_{Q R}^{P}\left(\Phi_{\lambda}(\zeta)\right) W_{\alpha}\left(\Phi^{Q}\right)(\lambda \zeta) W_{\bar{\alpha}}\left(\Phi^{R}\right)(\lambda \zeta)\right\} d \sigma_{1}(\zeta) \\
& \quad+2\left(\lambda^{2}-n\right) \int_{S^{2 n-1}} \Phi_{\lambda}^{P}(\zeta) N(\psi \circ W)(\lambda \zeta) d \sigma_{1}(\zeta) \\
& \quad+a_{n} \lambda^{2}\left(\lambda^{2}-1\right) \int_{S^{2 n-1}}\left\{\Phi_{\lambda}^{P}(\zeta)\left[T^{2}(\psi \circ W)(\lambda \zeta)+N^{2}(\psi \circ W)(\lambda \zeta)\right]\right. \\
& \left.\quad+4 \psi(\zeta) \Gamma_{Q R}^{P}\left(\Phi_{\lambda}(\zeta)\right) \xi\left(\Phi^{Q}\right)(\lambda \zeta) \bar{\xi}\left(\Phi^{R}\right)(\lambda \zeta)\right\} d \sigma_{1}(\zeta)=0
\end{aligned}
$$

and one has finally a manner to approach the boundary (since $\mathbb{B}_{n} \ni \lambda \zeta \rightarrow S^{2 n-1}$ as $\lambda \rightarrow 1^{-}$). The fact that the various parametric integrals in (7.29) have a limit as $\lambda \rightarrow 1^{-}$is proved in Lemmas 7.1 and 7.2, as a consequence of the basic Cimminolike assumption that $\Phi_{\lambda}^{P}$ has a limit in $\mathfrak{M}^{1}\left(S^{2 n-1}\right)$ as $\lambda \rightarrow 1^{-}$(of course a limit in $L^{2}\left(S^{2 n-1}\right)$ suffices for the linear part of the integrand in (7.29), $c f$. Lemma 7.1 below).

\section{Lemma 7.1.}

$$
\begin{aligned}
& \lim _{\lambda \rightarrow 1^{-}} \int_{S^{2 n-1}} \Phi_{\lambda}^{P}(\zeta) \Delta_{b}(\psi \circ W)(\lambda \zeta) d \sigma_{1}(\zeta)=\int_{S^{2 n-1}} \phi^{P}\left(\Delta_{b} \psi\right) d \sigma_{1}, \\
& \lim _{\lambda \rightarrow 1^{-}} \int_{S^{2 n-1}} \Phi_{\lambda}^{P}(\zeta) N(\psi \circ W)(\lambda \zeta) d \sigma_{1}(\zeta)=\frac{a_{n}-1}{a_{n}} \int_{S^{2 n-1}} \phi^{P} N(\psi) d \sigma_{1}, \\
& \lim _{\lambda \rightarrow 1^{-}} \int_{S^{2 n-1}} \Phi_{\lambda}^{P}(\zeta)\left[T^{2}(\psi \circ W)(\lambda \zeta)+N^{2}(\psi \circ W)(\lambda \zeta)\right] d \sigma_{1}(\zeta) \\
& =\int_{S^{2 n-1}} \phi^{P}\left[T^{2}(\psi)+\frac{2-a_{n}}{a_{n}^{2}} N(\psi)-\frac{1-a_{n}}{a_{n}} N^{2}(\psi)\right] d \sigma_{1} .
\end{aligned}
$$

Proof. One has

$$
X(\psi \circ W)_{\lambda \zeta}=\frac{1}{\lambda} X(\psi)_{\zeta}, \quad X^{2}(\psi \circ W)_{\lambda \zeta}=\frac{1}{\lambda^{2}} X^{2}(\psi)_{\zeta},
$$


for any $X \in T(\mathcal{F})$, hence

$$
\begin{aligned}
\Delta_{b}(\psi \circ W)(\lambda \zeta)= & -\frac{1}{\lambda^{2}} \sum_{\alpha}\left\{\left(W_{\alpha} W_{\bar{\alpha}} \psi\right)(\zeta)+\left(W_{\bar{\alpha}} W_{\alpha} \psi\right)(\zeta)\right. \\
& \left.-\lambda\left[\left(\nabla_{W_{\alpha}} W_{\bar{\alpha}}\right)(\psi)(\zeta)+\left(\nabla_{W_{\bar{\alpha}}} W_{\alpha}\right)(\psi)(\zeta)\right]\right\} .
\end{aligned}
$$

Then (7.30) is a consequence of

$$
\begin{aligned}
& \left|\int_{S^{2 n-1}} \Phi_{\lambda}^{P}(\zeta) \Delta_{b}(\psi \circ W)(\lambda \zeta) d \sigma_{1}(\zeta)-\int_{S^{2 n-1}} \phi^{P}(\zeta)\left(\Delta_{b} \psi\right)(\zeta) d \sigma_{1}(\zeta)\right| \\
& \leq \frac{1}{\lambda^{2}}\left\|\Phi_{\lambda}^{P}-\phi^{P}\right\|_{L^{2}\left(S^{2 n-1}\right)} \\
& \quad \times\left(\int _ { S ^ { 2 n - 1 } } \left[\sum_{\alpha=1}^{n}\left|\left(W_{\alpha} W_{\bar{\alpha}} \psi\right)(\zeta)+\left(W_{\bar{\alpha}} W_{\alpha} \psi\right)(\zeta)\right|\right.\right. \\
& \left.\left.\quad+\lambda \sum_{\alpha=1}^{n}\left|\left(\nabla_{W_{\alpha}} W_{\bar{\alpha}}\right)(\psi)(\zeta)+\left(\nabla_{W_{\bar{\alpha}}} W_{\alpha}\right)(\psi)(\zeta)\right|\right]^{2} d \sigma_{1}(\zeta)\right)^{\frac{1}{2}}+O(1-\lambda) .
\end{aligned}
$$

Similarly (7.31)-(7.32) follow from

$$
\begin{aligned}
N(\psi \circ W)_{\lambda \zeta} & =\frac{a_{n} \lambda-1}{a_{n} \lambda^{2}} N(\psi)_{\zeta} \\
N^{2}(\psi \circ W)_{\lambda \zeta} & =\frac{2-a_{n} \lambda}{a_{n}^{2} \lambda^{4}} N(\psi)_{\zeta}-\frac{1-a_{n} \lambda}{a_{n} \lambda^{2}} N^{2}(\psi)_{\zeta}
\end{aligned}
$$

and the estimates

$$
\begin{aligned}
& \left|\int_{S^{2 n-1}} \Phi_{\lambda}^{P}(\zeta) N(\psi \circ W)(\lambda \zeta) d \sigma_{1}(\zeta)-\frac{a_{n}-1}{a_{n}} \int_{S^{2 n-1}} \phi^{P} N(\psi) d \sigma_{1}\right| \\
& \leq \frac{a_{n} \lambda-1}{a_{n} \lambda^{2}}\left\|\Phi_{\lambda}^{P}-\phi^{P}\right\|_{L^{2}\left(S^{2 n-1}\right)}\|N(\psi)\|_{L^{2}\left(S^{2 n-1}\right)}+O(1-\lambda),
\end{aligned}
$$

and

$$
\begin{aligned}
& \mid \int_{S^{2 n-1}} \Phi_{\lambda}^{P}(\zeta)\left[T^{2}(\psi \circ W)(\lambda \zeta)+N^{2}(\psi \circ W)(\lambda \zeta)\right] d \sigma_{1}(\zeta) \\
& \quad-\int_{S^{2 n-1}} \phi^{P}\left[T^{2}(\psi)+\frac{2-a_{n}}{a_{n}^{2}} N(\psi)-\frac{1-a_{n}}{a_{n}} N^{2}(\psi)\right] d \sigma_{1} \mid \\
& \leq\left\{\frac{1}{\lambda}\left\|T^{2}(\psi)\right\|_{L^{2}\left(S^{2 n-1}\right)}+\frac{\left|2-a_{n} \lambda\right|}{a_{n}^{2} \lambda^{4}}\|N(\psi)\|_{L^{2}\left(S^{2 n-1}\right)}\right\}\left\|\Phi_{\lambda}^{P}-\phi^{P}\right\|_{L^{2}\left(S^{2 n-1}\right)}+O(1-\lambda) .
\end{aligned}
$$


Lemma 7.2. Let $\Phi \in W^{1}\left(\mathbb{B}_{n}, \mathbb{B}_{N}\right)$ be a Bergman-harmonic map into $\left(\mathbb{B}_{N}, h\right)$ with trace $\phi$ on $S^{2 n-1}$. Let us assume the following:

i) $\phi \in \mathfrak{M}^{1}\left(S^{2 n-1}, \mathbb{B}_{N}\right)$ i.e. $\phi \in \mathfrak{M}^{1}\left(S^{2 n-1}, \mathbb{R}^{2 N}\right)$ and $\phi(z) \in \mathbb{B}_{N}$ for a.e. $z \in \mathbb{B}_{n}$;

ii) $\lim _{\lambda \rightarrow 1^{-}} \Gamma_{Q R}^{P} \circ \Phi_{\lambda}=\Gamma_{Q R}^{P} \circ \phi$ in $L^{2}\left(S^{2 n-1}\right)$.

Then

$$
\begin{aligned}
& \lim _{\lambda \rightarrow 1^{-}} \int_{S^{2 n-1}}\left(\Gamma_{Q R}^{P} \circ \Phi_{\lambda}\right) W_{\alpha}\left(\Phi^{Q}\right)_{\lambda} W_{\bar{\alpha}}\left(\Phi^{R}\right)_{\lambda} d \sigma_{1} \\
& =\int_{S^{2 n-1}}\left(\Gamma_{Q R}^{P} \circ \phi\right) W_{\alpha}\left(\phi^{Q}\right) W_{\bar{\alpha}}\left(\phi^{R}\right) d \sigma_{1}
\end{aligned}
$$

for every $1 \leq P \leq 2 N$ and $1 \leq \alpha \leq n-1$.

Proof. Let $X \in H(\mathcal{F}), u \in W^{1}\left(\mathbb{B}_{n}\right)$ and $\psi \in C^{\infty}\left(S^{2 n-1}\right)$. Then for every $0<$ $\lambda<1$ and $v \geq 1$

$$
\begin{aligned}
& \int_{\mathbb{B}_{n}} X(u) \Psi_{\nu}^{\lambda} d \operatorname{vol}(g)=\int_{\epsilon_{0}-1 / v}^{\lambda+1 / v} t^{2 n-1} \chi_{v}^{\lambda}(t) d t \int_{S^{2 n-1}} X(u)(t \zeta) \psi(\zeta) d \sigma_{1}(\zeta) \\
& \rightarrow \int_{\epsilon_{0}}^{\lambda} t^{2 n-1} d t \int_{S^{2 n-1}} X(u)(t \zeta) \psi(\zeta) d \sigma_{1}(\zeta), \quad v \rightarrow \infty,
\end{aligned}
$$

and

$$
\begin{aligned}
& \int_{\mathbb{B}_{n}} X(u) \Psi_{v}^{\lambda} d \operatorname{vol}(g)=-\int_{\mathbb{B}_{n}} u \operatorname{div}_{g}\left(\Psi_{v}^{\lambda} X\right) d \operatorname{vol}(g) \\
& =-\int_{\epsilon_{0}-1 / v}^{\lambda+1 / v} t^{2 n-2} d t \int_{S^{2 n-1}} u_{t} X(\psi) d \sigma_{1}-\int_{\epsilon_{0}-1 / v}^{\lambda+1 / v} t^{2 n-1} \chi_{v}^{\lambda}(t) d t \int_{S^{2 n-1}} u_{t} \psi \operatorname{div}(X) d \sigma_{1} \\
& \rightarrow-\int_{\epsilon_{0}}^{\lambda} t^{2 n-1} d t \int_{S^{2 n-1}} u_{t}\left\{\frac{1}{t} X(\psi)+\psi \operatorname{div}(X)\right\} d \sigma_{1}, \quad v \rightarrow \infty .
\end{aligned}
$$

Hence

$$
\int_{\epsilon_{0}}^{\lambda} t^{2 n-1} d t \int_{S^{2 n-1}}\left\{X(u)_{t} \psi+u_{t}\left[\frac{1}{t} X(\psi)+\psi \operatorname{div}(X)\right]\right\} d \sigma_{1}=0
$$

and differentiation with respect to $\lambda$ (in the distribution sense) yields

$$
\int_{S^{2 n-1}} X(u)_{\lambda} \psi d \sigma_{1}=-\int_{S^{2 n-1}} u_{\lambda}\left[\frac{1}{\lambda} X(\psi)+\psi \operatorname{div}(X)\right] d \sigma_{1}
$$

for every $0<\lambda<1$. Subtraction of

$$
\int_{S^{2 n-1}} X\left(u_{\lambda}\right) \psi d \sigma_{1}=-\int_{S^{2 n-1}} u_{\lambda} \operatorname{div}(\psi X) d \sigma_{1}
$$


from (7.36) leads to

$$
X\left(u_{\lambda}\right)=\lambda X(u)_{\lambda}+(\lambda-1) \operatorname{div}(X) .
$$

Let us extend both sides of (7.37) by $\mathbb{C}$-linearity. Then (7.37) holds for every $X \in$ $H(\mathcal{F}) \otimes \mathbb{C}$. Next, by (7.37) with $X \in\left\{W_{\alpha}, W_{\bar{\alpha}}\right\}$,

$$
\begin{aligned}
& \int_{S^{2 n-1}}\left(\Gamma_{Q R}^{P} \circ \Phi_{\lambda}\right) W_{\alpha}\left(\Phi^{Q}\right)_{\lambda} W_{\bar{\alpha}}\left(\Phi^{R}\right)_{\lambda} d \sigma_{1} \\
& \quad-\int_{S^{2 n-1}}\left(\Gamma_{Q R}^{P} \circ \phi\right) W_{\alpha}\left(\phi^{Q}\right) W_{\bar{\alpha}}\left(\phi^{R}\right) d \sigma_{1} \\
& =\frac{1}{\lambda^{2}} \int_{S^{2 n-1}}\left(\Gamma_{Q R}^{P} \circ \Phi_{\lambda}-\Gamma_{Q R}^{P} \circ \phi\right)\left[W_{\alpha}\left(\Phi_{\lambda}^{Q}\right)-W_{\alpha}\left(\phi^{Q}\right)\right] \\
& \quad \times\left[W_{\bar{\alpha}}\left(\Phi_{\lambda}^{R}\right)-W_{\bar{\alpha}}\left(\phi^{R}\right)\right] d \sigma_{1} \\
& \quad+\frac{1}{\lambda^{2}} \int_{S^{2 n-1}}\left(\Gamma_{Q R}^{P} \circ \Phi_{\lambda}-\Gamma_{Q R}^{P} \circ \phi\right)\left\{\left[W_{\alpha}\left(\Phi_{\lambda}^{Q}\right)-W_{\alpha}\left(\phi^{Q}\right)\right] W_{\bar{\alpha}}\left(\phi^{R}\right)\right. \\
& \left.\quad+W_{\alpha}\left(\phi^{Q}\right)\left[W_{\bar{\alpha}}\left(\Phi_{\lambda}^{R}\right)-W_{\bar{\alpha}}\left(\phi^{R}\right)\right]\right\} d \sigma_{1} \\
& \quad+\frac{2}{\lambda^{2}} \int_{S^{2 n-1}}\left(\Gamma_{Q R}^{P} \circ \Phi_{\lambda}-\Gamma_{Q R}^{P} \circ \phi\right) W_{\alpha}\left(\phi^{Q}\right) W_{\bar{\alpha}}\left(\phi^{R}\right) d \sigma_{1}+O(1-\lambda)
\end{aligned}
$$

and

$$
\begin{aligned}
& \mid \int_{S^{2 n-1}}\left(\Gamma_{Q R}^{P} \circ \Phi_{\lambda}-\Gamma_{Q R}^{P} \circ \phi\right)\left[W_{\alpha}\left(\Phi_{\lambda}^{Q}\right)-W_{\alpha}\left(\phi^{Q}\right)\right] \\
& \quad \times\left[W_{\bar{\alpha}}\left(\Phi_{\lambda}^{R}\right)-W_{\bar{\alpha}}\left(\phi^{R}\right)\right] d \sigma_{1} \mid \\
& \leq \frac{1}{2}\left\|\Gamma_{Q R}^{P} \circ \Phi_{\lambda}-\Gamma_{Q R}^{P} \circ \phi\right\|_{L^{2}\left(S^{2 n-1}\right)} \\
& \quad \times\left\{\left\|W_{\alpha}\left(\Phi_{\lambda}^{Q}\right)-W_{\alpha}\left(\phi^{Q}\right)\right\|_{L^{2}\left(S^{2 n-1}\right)}+\left\|W_{\bar{\alpha}}\left(\Phi_{\lambda}^{R}\right)-W_{\bar{\alpha}}\left(\phi^{R}\right)\right\|_{L^{2}\left(S^{2 n-1}\right)}\right\} .
\end{aligned}
$$

Lemma 7.2 is proved. Statement (a) in Theorem 1.3 follows from Lemmas 7.1 and 7.2 by taking the limit as $\lambda \rightarrow 1^{-}$in (7.29).

To prove statement (b) one sums (7.28) for $P=A$ to (7.28) for $P=A+N$ multiplied by $i=\sqrt{-1}$ so to obtain, by (6.19),

$$
\begin{aligned}
& \left(\lambda^{2}-1\right) \int_{S^{2 n-1}} F_{\lambda}^{A}\left\{\Delta_{b}(\psi \circ W)_{\lambda}+2\left(\lambda^{2}-n\right) N(\psi \circ W)_{\lambda}\right. \\
& \left.+a_{n} \lambda^{2}\left(\lambda^{2}-1\right)\left[T^{2}(\psi \circ W)_{\lambda}+N^{2}(\psi \circ W)_{\lambda}\right]\right\} d \sigma_{1} \\
& +\frac{2(n+1)}{a_{n}} \int_{S^{2 n-1}} \psi \gamma^{A}(\Phi)_{\lambda} d \sigma_{1}=0 .
\end{aligned}
$$


Identity (6.21) for $\Omega=\mathbb{B}_{n}$ gives

$$
\begin{aligned}
& \frac{n+1}{a_{n}} \gamma^{A}(\Phi)_{\lambda}=\frac{\lambda^{2}-1}{\left|F_{\lambda}\right|^{2}-1}\left[\delta_{B}^{A}\left(F_{\lambda}\right)_{\bar{C}}+\delta_{C}^{A}\left(F_{\lambda}\right)_{\bar{B}}\right] \\
& \quad \times\left[\sum_{\alpha=1}^{n-1} W_{\alpha}\left(F^{B}\right)_{\lambda} W_{\bar{\alpha}}\left(F^{C}\right)_{\lambda}-2 a_{n} \lambda^{2}\left(\lambda^{2}-1\right) \xi\left(F^{B}\right)_{\lambda} \bar{\xi}\left(F^{C}\right)_{\lambda}\right] .
\end{aligned}
$$

Note that (7.38)-(7.39) are the $L^{2}$ analogs to the partial differential equations (6.20)(6.21). In the pointwise version of (7.38) one may (after substitution from (7.39)) i) simplify $\lambda^{2}-1$, ii) eliminate $\left|F_{\lambda}(\zeta)\right|^{2}-1$, and iii) approach the boundary with $\lambda \rightarrow 1^{-}$. A different kind of asymptotic analysis should be conducted in the case at hand (because $F_{\lambda}(\zeta)$ depends on the variable of integration). This prompts our assumption on the existence of the limit (1.6) and $L^{2}$ convergence is seen to suffice. A remark on the nature of our hypothesis (v) in Theorem 1.3 is in order. If $Q_{\Phi}$ is the quotient defined by (6.22) with $\Omega=\mathbb{B}_{n}$ and $D=\mathbb{B}_{N}$ then $\left(Q_{\Phi}\right)_{\lambda}=\left(1-\left|F_{\lambda}\right|^{2}\right) /\left(1-\lambda^{2}\right)$ is assumed to have a $L^{2}$ limit $Q_{\phi} \in L^{2}\left(S^{2 n-1}\right)$ as $\lambda \rightarrow 1^{-}$. To give an example, each proper holomorphic map $\Phi: \mathbb{B}_{2} \rightarrow \mathbb{B}_{3}$ in Faran's list ( $c f$. [16]) has this property.

\begin{tabular}{|c|c|}
\hline$\Phi(z, w),(z, w) \in \mathbb{B}_{2}$ & $Q_{\phi}(z, w),(z, w) \in S^{3}$ \\
\hline$\left(z^{3}, \sqrt{3} z w, w^{3}\right)$ & $3\left(1-|z|^{2}|w|^{2}\right)$ \\
\hline$\left(z^{2}, \sqrt{2} z w, w^{2}\right)$ & 2 \\
\hline$\left(z, z w, w^{2}\right)$ & $1+|w|^{2}$ \\
\hline$(0, z, w)$ & 1 \\
\hline
\end{tabular}

Assumptions such as the existence of $\lim _{z \rightarrow S^{2 n-1}}(1-|\Phi(z)|) /(1-|z|)-$ in our context $\lim _{\lambda \rightarrow 1^{-}} A(\lambda)$ where $A(\lambda)=\left(1-\left|F_{\lambda}\right|\right) /(1-\lambda)$ - proved useful in existence results for non-tangential limits $\lim _{z \rightarrow S^{2 n-1}} \Phi(z), c f$. e.g. [30] (cf. also [1] for generalizations to maps of strictly pseudoconvex domains). Here $\left(Q_{\Phi}\right)_{\lambda}=$ $A(\lambda) B(\lambda)$ and $L^{2}-\lim _{\lambda \rightarrow 1^{-}} \Phi_{\lambda}=\phi$ yields i) $L^{2}-\lim _{\lambda \rightarrow 1^{-}} B(\lambda)=1$ and ii) $L^{2}$ $\lim _{\lambda \rightarrow 1^{-}}\left(Q_{\Phi}\right)_{\lambda}$ exists if and only if $L^{2}-\lim _{\lambda \rightarrow 1^{-}} A(\lambda)$ exists. Let $\theta_{0}=\frac{i}{2}(\bar{\partial}-\partial)(z \bar{z}+$ $w \bar{w})$ and $\Theta_{0}=\frac{i}{2}(\bar{\partial}-\partial)|Z|^{2}, Z=\left(Z_{1}, Z_{2}, Z_{3}\right)$, be the canonical contact forms on $S^{3}$ and $S^{5}$. Let $\phi: S^{3} \rightarrow S^{5}$ be the boundary values of $\Phi \in P(2,3)$. The dilation $\lambda(\phi)=\lambda\left(\phi ; \theta_{0}, \Theta_{0}\right) \in C^{\infty}\left(S^{3}, \mathbb{R}\right)$ is given by $\phi^{*} \Theta_{0}=\lambda(\phi) \theta_{0}$. A parallel 
of the table above to calculations in [5] shows that $Q_{\phi}=\lambda(\phi)$ for every map in Faran's list. This is a curious phenomenon, lacking an explanation as yet, relating the dilation of $\phi$ (a quantity of metric nature) to the quotient $Q_{\phi}$ (accounting for regularity of sorts $e . g$. existence of angular derivatives).

Going back to the proof of (b) in Theorem 1.3 let us replace from (7.39) into (7.38) and take the limit as $\lambda \rightarrow 1^{-}$. We obtain

$$
\int_{S^{2 n-1}} \psi Q_{\phi}^{-1}\left(\delta_{B}^{A} \phi_{\bar{B}}+\delta_{C}^{A} \phi_{\bar{B}}\right) \sum_{\alpha} W_{\alpha}\left(\phi^{B}\right) W_{\bar{\alpha}}\left(\phi^{C}\right) d \sigma_{1}=0
$$

for every $\psi \in C^{\infty}\left(S^{2 n-1}\right)$. Let $A \in\{1, \ldots, N\}$ be a fixed index. As $\phi_{\bar{A}} \in$ $L^{2}\left(S^{2 n-1}\right)$ there is a sequence $\left\{\psi_{\nu}\right\}_{\nu \geq 1} \subset C^{\infty}\left(S^{2 n-1}\right)$ such that $\lim _{\nu \rightarrow \infty} \psi_{\nu}=\phi_{\bar{A}}$ in $L^{2}\left(S^{2 n-1}\right)$. Then

$$
\begin{aligned}
0 & =\int_{S^{2 n-1}} \psi_{\nu} Q_{\phi}^{-1}\left(\delta_{B}^{A} \phi_{\bar{B}}+\delta_{C}^{A} \phi_{\bar{B}}\right) \sum_{\alpha} W_{\alpha}\left(\phi^{B}\right) W_{\bar{\alpha}}\left(\phi^{C}\right) d \sigma_{1} \\
& \rightarrow \int_{S^{2 n-1}} Q_{\phi}^{-1} \phi_{\bar{A}}\left(\delta_{B}^{A} \phi_{\bar{B}}+\delta_{C}^{A} \phi_{\bar{B}}\right) \sum_{\alpha} W_{\alpha}\left(\phi^{B}\right) W_{\bar{\alpha}}\left(\phi^{C}\right) d \sigma_{1}, \quad v \rightarrow \infty,
\end{aligned}
$$

and contraction over $A$ yields

$$
\int_{S^{2 n-1}} Q_{\phi}^{-1} \phi_{\bar{B}} \phi_{\bar{C}} \sum_{\alpha} W_{\alpha}\left(\phi^{B}\right) W_{\bar{\alpha}}\left(\phi^{C}\right) d \sigma_{1}=0 .
$$

By Lemma 2.1 and the constraint $\phi_{\bar{A}} \phi^{A}=1$ one has $\phi_{\bar{C}} W_{\bar{\alpha}}\left(\phi^{C}\right)=-\phi^{C} W_{\bar{\alpha}}\left(\phi_{\bar{C}}\right)$, hence

$$
\int_{S^{2 n-1}} Q_{\phi}^{-1} \sum_{\alpha}\left|W_{\bar{\alpha}}\left(\phi_{\bar{C}}\right) \phi^{C}\right|^{2} d \sigma_{1}=0 .
$$

Finally (as $Q_{\phi}>0$ a.e. on $S^{2 n-1}$ ) $W_{\bar{\alpha}}\left(\phi_{\bar{C}}\right) \phi^{C}=0$ a.e. on $S^{2 n-1}$ for every $1 \leq$ $\alpha \leq n-1$.

Let us prove (c) in Teorem 1.3. If $\phi: S^{2 n-1} \rightarrow S^{2 N-1}$ is a weakly CR map then each $\phi^{A}$ is a weakly CR function i.e.

$$
\int_{S^{2 n-1}} \phi^{A} \operatorname{div}(\bar{Z}) d \sigma_{1}=0, \quad Z \in C^{\infty}\left(T_{1,0}\left(S^{2 n-1}\right)\right) .
$$

Let $\left\{T_{\alpha}: 1 \leq \alpha \leq n-1\right\}$ be a local $G_{\eta}$-orthonormal frame of $T_{1,0}\left(S^{2 n-1}\right)$, defined on the open set $U \subset S^{2 n-1}$. Then for every $\psi \in C_{0}^{\infty}(U)$ and every sequence $\left\{\psi_{v}\right\}_{v \geq 1} \subset C_{0}^{\infty}(U)$ such that $\lim _{v \rightarrow \infty} \psi_{v}=\psi \phi_{\bar{A}}$ in $L^{2}(U)$

$$
\begin{aligned}
0 & =\int_{U} \phi^{A} \operatorname{div}\left(\psi_{\nu} T_{\bar{\alpha}}\right) d \sigma_{1}=-\int_{U} G_{\eta}\left(\nabla^{H} \phi^{A}, \psi_{\nu} T_{\bar{\alpha}}\right) d \sigma_{1} \\
& \rightarrow \int_{U} \psi \phi_{\bar{A}} T_{\bar{\alpha}}\left(\phi^{A}\right) d \sigma_{1}, \quad \nu \rightarrow \infty .
\end{aligned}
$$


At this point we may contract over $A$ and replace $\psi$ by $\varphi_{v} T_{\alpha}\left(\bar{\varphi}_{\nu}\right)$ where $\left\{\varphi_{\nu}\right\}_{\nu \geq 1} \subset$ $C_{0}^{\infty}(U)$ is chosen such that $\varphi_{v} \rightarrow \phi^{B}$ in $L^{2}(U)$ as $v \rightarrow \infty$, with $B \in\{1, \ldots, N\}$ fixed. One has

$$
\begin{aligned}
0 & =\int_{U} \varphi_{\nu} T_{\alpha}\left(\bar{\varphi}_{\nu}\right) \phi_{\bar{A}} T_{\bar{\alpha}}\left(\phi^{A}\right) d \sigma_{1} \\
& \rightarrow \int_{U} \phi^{B} T_{\alpha}\left(\phi_{\bar{B}}\right) \phi_{\bar{A}} T_{\bar{\alpha}}\left(\phi^{A}\right) d \sigma_{1}, \quad v \rightarrow \infty,
\end{aligned}
$$

so that, by contraction over $B, \int_{U}\left|\phi_{\bar{A}} T_{\bar{\alpha}}\left(\phi^{A}\right)\right|^{2} d \sigma_{1}=0$ i.e. $\phi_{\bar{A}} T_{\bar{\alpha}}\left(\phi^{A}\right)=0$ a.e. in $U$.

\section{References}

[1] M. Aв BTE, The Lindelöf principle and the angular derivative in strongly convex domains, J. Analyse Math. 54 (1990), 189-228; Angular derivatives in strongly pseudoconvex domains, Proc. Sympos Pure Math. 52, Part 2 (1991), 23-40.

[2] E. BARLETTA, On the boundary behavior of the holomorphic sectional curvature of the Bergman metric, Matematiche 61 (2006), 301-316.

[3] E. BARLeTta, S. Dragomir and H. URAKAWA, Pseudoharmonic maps from a nondegenerate CR manifold into a Riemannian manifold, Indiana Univ. Math. J. 50 (2001), 719-746.

[4] E. Barletta, S. Dragomir and H. URAKawa, Yang-Mills fields on CR manifolds, J. Math. Phys. 47 (2006), 41 pp.

[5] E. BARletta and S. DRAGOMIR, Proper holomorphic maps in harmonic map theory, Ann. Mat. Pura Appl. 194 (2015), 1469-1498.

[6] A. Bonfiglioli and E. LANCONelli, Dirichlet problem with $L^{p}$-boundary data in contractible domains of Carnot groups, Ann. Sc. Norm. Sup. Pisa Cl. Sci. 5 (2006), 579-610.

[7] A. Bonfiglioli, E. Lanconelli and F. Uguzzoni, "Stratified Lie Groups and Potential Theory for their Sub-Laplacians", Springer Monographs in Math., Springer-Verlag, BerlinHeidelberg, 2007.

[8] L. CAPOGNA and N. Garofalo, Regularity of minimizers of the calculus of variations in Carnot groups via hypoellipticity of systems of Hörmander type, J. Eur. Math. Soc. (JEMS) 5 (2003), 1140.

[9] G. Cimmino, Nuovo tipo di condizione al contorno e nuovo metodo di trattazione per il problema generalizzato di Dirichlet, Rend. Circ. Mat. Palermo 61 (1937), 177-221.

[10] G. Cimmino, "Opere Scelte", a cura di Carlo Sbordone e Guido Trombetti, Accademia di Scienze Fisiche e Matematiche della Società Nazionale di Scienze Lettere e Arti in Napoli, Giannini, Napoli, 2002.

[11] J. D'Angelo, Polynomial proper maps between balls, Duke Math. J. 57 (1988), 211-219.

[12] J. D'ANGELO, Proper holomorphic maps between balls of different dimensions, Michigan Math. J. 35 (1988), 83-90.

[13] J. D’Angelo, Polynomial proper holomorphic mappings between balls, II, Michigan Math. J. 38 (1991), 53-65.

[14] S. Dragomir and S. NishikaWA, Foliated CR manifolds, J. Math. Soc. Japan 56 (2004), 1031-1068.

[15] S. DRAGOMIR and G. Tomassini, "Differential Geometry and Analysis on CR Manifolds", Progress in Mathematics, Vol. 246, Birkhäuser, Boston-Basel-Berlin, 2006.

[16] J. FARAN, Maps from the 2 ball to the 3 ball, Invent. Math. 68 (1982), 441-475.

[17] C. FEFFERMAN, The Bergman kernel and biholomorphic equivalence of pseudoconvex domains, Invent. Math. 26 (1974), 1-65. 
[18] D. Gilbarg and N. S. Trudinger, "Elliptic partial differential equations of second order", Grundl. Math. Wiss., Vol. 224, Springer-Verlag, Berlin-Heidelberg-New York-Tokyo, 1983.

[19] C. R. Graham and J. M. LeE, Smooth solutions of degenerate Laplacians on strictly pseudoconvex domains, Duke Math. J. 57 (1988), 697-720.

[20] C. R. Graham, The Dirichlet problem for the Bergman laplacian. I, Comm. Partial Differential Equations 8 (1983), 433-476.

[21] C. R. Graham, The Dirichlet problem for the Bergman laplacian. II, Comm. Partial Differential Equations 8 (1983), 563-641.

[22] J. Jost, "Harmonic Mappings Between Riemannian Manifolds", Proceedings of the Center for Mathematical Analysis, Australian National University, Vol. 4, 1983.

[23] J. Jost and C-J. XU, Subelliptic harmonic maps, Trans. Amer. Math. Soc. 350 (1998), 4633-4649.

[24] P. F. KLEMBECK, Kähler metrics of negative curvature, the Bergman metric near the boundary, and the Kobayashi metric on smooth bounded strictly pseudoconvex sets, Indiana Univ. Math. J. 27 (1978), 275-282.

[25] A. KoRÁNYI and H. M. REIMANn, Contact transformations as limits of symplectomorphisms, C.R. Acad. Sci. Paris 318 (1994), 1119-1124.

[26] E. Lanconelli, Dirichlet problem with $L^{p}$-boundary data for real sub-Laplacians, Matematiche 60 (2005), 269-277.

[27] J. M. LEE and R. MeLROSE, Boundary behaviour of the complex Monge-Ampère equation, Acta Math. 148 (1982), 159-192.

[28] A. Lichnerowicz, "Applications harmoniques de variétés Kaehleriennes", Symposia Mathematica, Vol. III, Bologna, 1970.

[29] R. Moser, "Partial Regularity for Harmonic Maps and Related Problems", World Scientific, New Jersey-London-Singapore-Beijing-Shanghai-Hong Kong-Taipei-Chennai, 2005.

[30] W. Rudin, "Function Theory in the Unit ball of $\mathbb{C}^{n}$ ", Springer-Verlag, New YorkHeidelberg-Berlin, 1980.

[31] C. WANG, Subelliptic harmonic maps from Carnot groups, Calc. Var. 18 (2003), 95-115.

[32] S. M. WeBSTER, The rigidity of $C-R$ hypersurfaces in a sphere, Indiana Univ. Math. J. 28 (1979), 405-416.

[33] S. M. Webster, Pseudohermitian structures on a real hypersurface, J. Differential Geom. 13 (1978), 25-41.

[34] C-J. XU, Subelliptic variational problems, Bull. Soc. Math France 118 (1990), 147-169.

[35] C-J. XU and C. ZUILY, Higher interior regularity for quasilinear subelliptic systems, Calc. Var. 5 (1997), 323-343.

[36] Z-R. ZHOU, Uniqueness of subelliptic harmonic maps, Ann. Glob. Anal. Geom. 17 (1999), 581-594.

[37] Z-R. ZHOU, Heat flows of subelliptic harmonic maps into Riemannian manifolds with nonpositive curvatures, J. Geom. Anal. 23 (2013), 471-489.

Università degli Studi della Basilicata

Dipartimento di Matematica, Informatica ed Economia Via dell'Ateneo Lucano, 10

85100 Potenza, Italia

elisabetta.barletta@unibas.it

sorin.dragomir@unibas.it 